\title{
Aquatische Ökosysteme: Nordsee, Wattenmeer, Elbeästuar und Ostsee
}

Justus van Beusekom, Ralf Thiel, Ivo Bobsien, Maarten Boersma, Christian Buschbaum, Andreas Dänhardt, Alexander Darr, René Friedland, Matthias Kloppmann, Ingrid Kröncke, Johannes Rick, Markus Wetzel

5.1 Einleitung - 90

5.2 Nordsee - 90

5.2.1 Plankton - 90

5.2.2 Makrozoobenthos - 91

5.2.3 Fische -92

5.3 Wattenmeer - 93

5.3.1 Plankton - 94

5.3.2 Makrozoobenthos - 94

5.3.3 Fische -95

5.4 Elbeästuar - 96

5.4.1 Plankton - 96

5.4.2 Makrozoobenthos - 96

5.4.3 Fische -98

5.5 Lübecker Bucht - 98

5.5.1 Plankton - 100

5.5.2 Benthos - 101

5.5.3 Fische - 102

$5.6 \quad$ Fazit -102

Literatur - 103 


\subsection{Einleitung}

Langzeitbeobachtungen von aquatischen Ökosystemen zeigen nicht nur eine hohe Variabilität, sondern auch graduelle Änderungen und sog. Regimeshifts: sprunghafte Änderungen im Funktionieren des gesamten Ökosystems. Sowohl die natürliche Variabilität hydrodynamischer und atmosphärischer Prozesse auf verschiedenen Zeitskalen als auch menschliche Einflussnahmen wie beispielsweise $\mathrm{CO}_{2}$-Ausstoß, Fischerei, Deichbau, Vertiefungen oder Eutrophierung tragen zu dieser komplexen ökologischen Dynamik bei (z. B. Emeis et al. 2015).

Im 1. Klimabericht für die Metropolregion Hamburg (1. HKB; Colijn und Fanger 2011) wurde die Klimaauswirkung auf das Phyto- und Zooplankton, das Makrozoobenthos und die Fische in Nordsee, Wattenmeer und Elbeästuar dargelegt. Die Autoren zeigten, dass insbesondere die Wassertemperatur und Hydrodynamik die Variabilität der Ökosysteme prägten, während hin zum Wattenmeer und Elbeästuar die Interaktion mit anthropogenen Faktoren zunahm und vermutlich die zukünftige ökologische Entwicklung prägen wird.

In der Nordsee traten abrupte Änderungen vor allem am Ende der 1980er-Jahre auf, die zu einem deutlichen Regimeshift geführt haben. Wärmeliebende und eingeschleppte Arten breiteten sich aus. Das Ausbleiben kalter Winter prägte die Entwicklung von Makrozoobenthos, Fischfauna und Plankton, Letzteres durch eine Zunahme des Fraßdrucks von Copepoden (Ruderfußkrebsen) nach warmen Wintern. Eine zunehmende Dominanz von Quallen und negative Effekte der Versauerung auf die Planktongemeinschaft wurden erwartet.

Auch im Wattenmeer prägten höhere Temperaturen sowohl im Winter als auch im Sommer die Langzeitdynamik. Die Wechselwirkung von natürlichen und anthropogenen Faktoren wurde hervorgehoben. Die Temperaturerhöhung hat zu einer verstärkten Ausbreitung eingeschleppter Arten geführt. Insbesondere hat die nichtheimische pazifische Auster das Ökosystem des Wattenmeeres grundlegend verändert. Im Gegensatz zur Nordsee nahmen im Pelagial die Copepoden zu. Für die Zukunft wurde erwartet, dass insbesondere der Meeresspiegelanstieg (und als Folge ein verstärkter Deichbau) das Wattenmeer prägen wird. Offen ist die Frage, ob Wattflächen trotz Meeresspiegelanstiegs durch Sedimentationsprozesse in vergleichbarer Ausdehnung wie bisher erhalten bleiben.

Das Elbeästuar wurde vor allem durch menschliche Einflussnahmen wie Eindeichung und Baggerung geprägt. Die ökologische Auswirkung einer Klimaänderung wird in hohem Maße vom Ausmaß der strombaulichen Maßnahmen abhängen. Mit weiteren Verlusten von Flachwassergebieten, Wattflächen und Vordeichland und den damit zusammenhängenden ökologischen Werten sollte gerechnet werden. Eine Verstärkung der bereits auftretenden Sauerstoffdefizite im Zusammenhang mit einer Verlagerung der oberen Brackwassergrenze stromaufwärts wurde als wahrscheinlich erachtet.

Colijn und Fanger (2011) hoben hervor, dass Vorsicht geboten ist bei der Extrapolation in die Zukunft der o. g., auf der Variabilität des Systems basierenden Trends. Interaktive Prozesse zwischen den klimabedingten Änderungen und anthropogen bedingten Veränderungen sind schwer vorhersagbar.
In diesem Kapitel werden neue Erkenntnisse zu klimabedingten Änderungen in aquatischen Ökosystemen mit Bezug zur Metropolregion Hamburg zusammengefasst und die Aussagen aus dem 1. HKB ggf. kritisch beleuchtet. Neu ist hierbei die ökologische Betrachtung der Lübecker Bucht.

\subsection{Nordsee}

Die Nordsee ist ein flaches Schelfmeer, das sowohl durch natürliche Variabilität als auch durch menschliche Faktoren beeinflusst wird (Emeis et al. 2015). Menschliche Eingriffe fingen vor etwa 1000 Jahren mit Eindeichungen an, beschleunigten sich in den 1950er-Jahren und erstreckten sich z. B. durch Windkraftanlagen und Fischerei immer weiter in die offene Nordsee hinein. Darüber hinaus hat sich die Nordsee während der letzten Jahrzehnte deutlich erwärmt (s. auch Abschn. 4.1). Die Analyse ökologischer Langzeitdaten zeigt die überragende Bedeutung des Temperaturregimes z. B. durch die prägende Auswirkung von extrem kalten Wintern auf das Überleben bestimmter Arten, durch die Etablierung eingeschleppter Arten infolge wärmerer Sommer oder durch eine geänderte Schichtung der Nordsee. Letzteres bezieht sich auf die Ausbildung einer leichteren, wärmeren Oberflächenschicht im Sommer durch die erhöhte Sonneneinstrahlung und geringere Durchmischung. Es ist nach wie vor schwierig, anthropogene und natürliche Faktoren zu trennen (Emeis et al. 2015).

\subsubsection{Plankton}

Das Phytoplankton steht an der Basis der Nahrungsketten, und jegliche Änderungen können das gesamte Ökosystem beeinflussen. Licht, Nährstoffe und Wegfraß bestimmen die Wachstumsmöglichkeiten des Phytoplanktons. Die Ausbildung einer Schichtung führt z. B. dazu, dass das Phytoplankton mehr Licht zur Verfügung hat und dadurch zunächst besser wachsen kann, bis die Nährstoffe aufgebraucht sind. Winder und Sommer (2012) fassten den Stand des Wissens zusammen und zeigten, dass eine Klimaerwärmung in erster Linie zu einer verstärkten Schichtung und dadurch zu einem geringeren Nährstofffluss in die obere Wasserschicht führen wird. Die dadurch verursachte stärkere Nährstofflimitierung wird zu kleineren Phytoplanktonarten führen. Wärmere Temperaturen werden die maximale Photosynthese steigern. Weil erhöhte Temperaturen die heterotrophen Prozesse beschleunigen, wird der Fraßdruck zunehmen. Phytoplankton und Zooplankton reagieren zudem oft unterschiedlich auf Änderungen der Umweltbedingungen. Dies kann zur Veränderung der Planktonzusammensetzung und den damit verknüpften Nahrungsbeziehungen führen (Mackas et al. 2012).

Es ist zu erwarten, dass die ansteigende Kohlendioxidkonzentration durch Veränderungen im Karbonatsystem vor allem das kalzifizierende Phytoplankton (vor allem Coccolithophoriden) beeinflusst (IPCC 2014). Beaugrand et al. (2013) zeigten aber, dass die Langzeitvariabilität des kalzifizierenden Planktons noch nicht durch $\mathrm{pH}$-Regime, sondern durch Temperatur gesteuert wird. 
Seit den 1960er-Jahren gewinnt das Phytoplankton bei Helgoland nun früher im Jahr an Bedeutung (Wiltshire et al. 2015; - Abb. 5.1). Lohmann und Wiltshire (2012) fanden eine enge Verknüpfung des Beginns der Diatomeenfrühjahrsblüten mit der winterlichen Atmosphärenzirkulation. Erlaubte die Zirkulation über Skandinavien einen vermehrten Einstrom von klarem, mehr marinem und wärmerem atlantischem Wasser in die Nordsee, so trat die Blüte eher auf. Sie erfolgte später, wenn eine mehr kontinental geprägte Zirkulation aus dem Osten vorherrschte. Tian et al. (2011) zeigten, dass in Jahren, in denen relativ trübes Küstenwasser dominierte, die Frühjahrsblüte noch vor der Entwicklung einer Schichtung begann und von der mittleren Lichtverfügbarkeit in der Wassersäule abhängig war (s. auch Scharfe 2013). Dominierte hingegen mehr küstenfernes Nordseewasser, folgte die Frühjahrsblüte eher der Ausbildung einer Schichtung und erschien weniger abhängig von der Lichtverfügbarkeit im Wasser.

Schlüter et al. (2012) untersuchten die Saisonalität dreier bestandsbildender Diatomeen seit den 1960er-Jahren. Während bei Guinardia delicatula und Odontella aurita ein signifikanter Trend hin zu früheren Blütezeitpunkten festgestellt werden konnte, zeigte Thalassionema nitzschioides einen ebenfalls signifikanten Trend hin zu einem früheren Zusammenbrechen der Blüte. Zeitgleich zum oft zitierten Regimeshift in der Nordsee Ende der 1980erJahre finden sich z. T. Ausweitungen artspezifischer Nischen von planktischen (z. B. Skeletonema sp.) und tychopelagischen (Paralia sulcata) Diatomeen (Freund et al. 2012; Gebühr et al. 2009).

Inwieweit das erstmalige Auftreten der großen, sehr auffälligen Kieselalge Mediopyxis helysia in der Deutschen Bucht mit der Temperaturerhöhung zusammenhängt, ist momentan noch unklar. Die Art wurde 2003 zunächst in der Sylt-Rømø-Bucht und dann 2009 auch bei Helgoland nachgewiesen (Kraberg et al. 2012). Sie entwickelte sich zunächst zu einem wichtigen Vertreter des Phytoplanktons der Deutschen Bucht mit maximalen Häufigkeiten von > 300.000 Zellen/l. Seit 2012 sind die maximalen Biomassen dieser neuen Art allerdings wieder deutlich rückläufig (Kraberg et al. 2012; Rick et al. 2015).

In diesem Jahrhundert hat sich die Erhöhung der Diatomeendichten und eine Verringerung der Dichten der Dinoflagellaten weiter durchgesetzt (Wiltshire et al. 2010; Boersma et al. 2015). Da Dinoflagellaten eine sehr wichtige Nahrungsquelle für neritische Copepoden darstellen (Gentsch et al. 2009), hat sich die Nahrungssituation für die Copepoden verschlechtert (AlvarezFernandez et al. 2012), was potenziell zu einer Verringerung der Dichten der herbivoren und omnivoren Copepoden geführt hat (Boersma et al. 2015). Zudem hat durch die abnehmenden Phosphoreinträge in die Deutsche Bucht die Qualität der Algen als Futter für Copepoden (Malzahn und Boersma 2012) wahrscheinlich abgenommen. Tatsächlich korreliert die Copepodendichte mit der Gesamtmenge an Phosphor (Boersma et al. 2015). Eine weitere Abnahme der Phosphorfrachten in die Deutsche Bucht könnte Auswirkungen auf höhere trophische Ebenen haben.

Wie das Phytoplankton entwickeln sich auch die Copepoden fast fünf Wochen früher als noch vor 40 Jahren (Wiltshire und Boersma 2016; $D$ Abb. 5.1). Während das Phytoplankton vor allem auf die ständig steigende Transparenz des Wassers reagiert (s. o.), steuern die Frühjahrstemperaturen das Zooplankton (Wiltshire und Boersma 2016, $\mathbf{D}$ Abb. 5.1). Es ist daher vielleicht

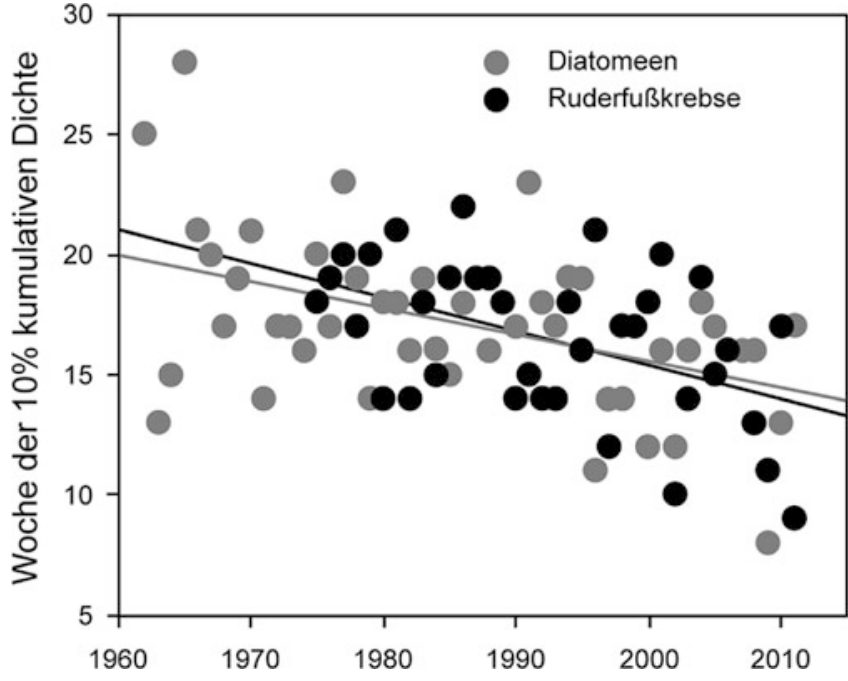

- Abb. 5.1 Woche, in der für Diatomeen und Ruderfusskrebse10 \% der jährlichen kumulativen Dichte erreicht werden. Die Änderungen in diesem Maß für den Anfang der Wachstumsphase in jedem Jahr sind für beide Gruppen identisch. (Wiltshire und Boersma 2016)

ein Zufall, dass es in der Deutschen Bucht noch nicht zu einem Mismatch zwischen Angebot und Nachfrage von Nahrung für die Copepoden gekommen ist.

Im letzten Klimabericht wurde über das an Bedeutung gewinnende gelatinöse Zooplankton (Quallen) berichtet. Die damals gerade eingewanderte Rippenqualle Mnemiopsis leidyi hat sich scheinbar ohne negative Folgen im Ökosystem etabliert. Die im Vergleich zu Fischen höhere Toleranz vieler Quallen gegenüber den mit dem globalen Wandel zusammenhängenden Faktoren Sauerstoffmangel, Wassertemperatur und pH-Stress (z. B. Gambill und Peck 2014) kann dazu führen, dass sie als Gewinner der globalen Wandlungsprozesse hervortreten.

\subsubsection{Makrozoobenthos}

Neumann et al. (2013b) analysierten und verglichen die räumliche Verbreitung der Gemeinschaften von Fischen sowie In- und Epifauna in der deutschen Ausschließlichen Wirtschaftszone (AWZ), um darauf basierend benthische Habitate zu identifizieren. Es wurden drei Habitate identifiziert: die Küstenzone, der Oyster Ground und der Entenschnabel der Doggerbank, die mit spezifischen Umweltfaktoren und deren saisonaler Variabilität korreliert sind. Trotz Temperaturanstieg sind die Habitate bislang stabil. Dennoch zeigen Langzeituntersuchungen der Epifauna-Gemeinschaften seit 1998 in der südöstlichen Nordsee, dass besonders die küstennahen Gebiete stärker von einem Temperaturanstieg beeinflusst wurden (Neumann et al. 2009) und wahrscheinlich auch weiterhin werden.

Seit 1978 werden die Infauna-Gemeinschaften im Inselvorfeld von Norderney untersucht und zeigten über zwei Jahnzehnte eine hohe Korrelation mit dem Nordatlantischen Oszillationsindex (NAOI). Dieser Zusammenhang existiert seit 2001 nicht mehr, da die NAOI ihre Persistenz und ihre Autokorrelation verloren hat und deshalb kein guter Klimaprädiktor ist (Dippner et al. 2014). Ausgangspunkt war 2000/2001 ein nichtlinearer 

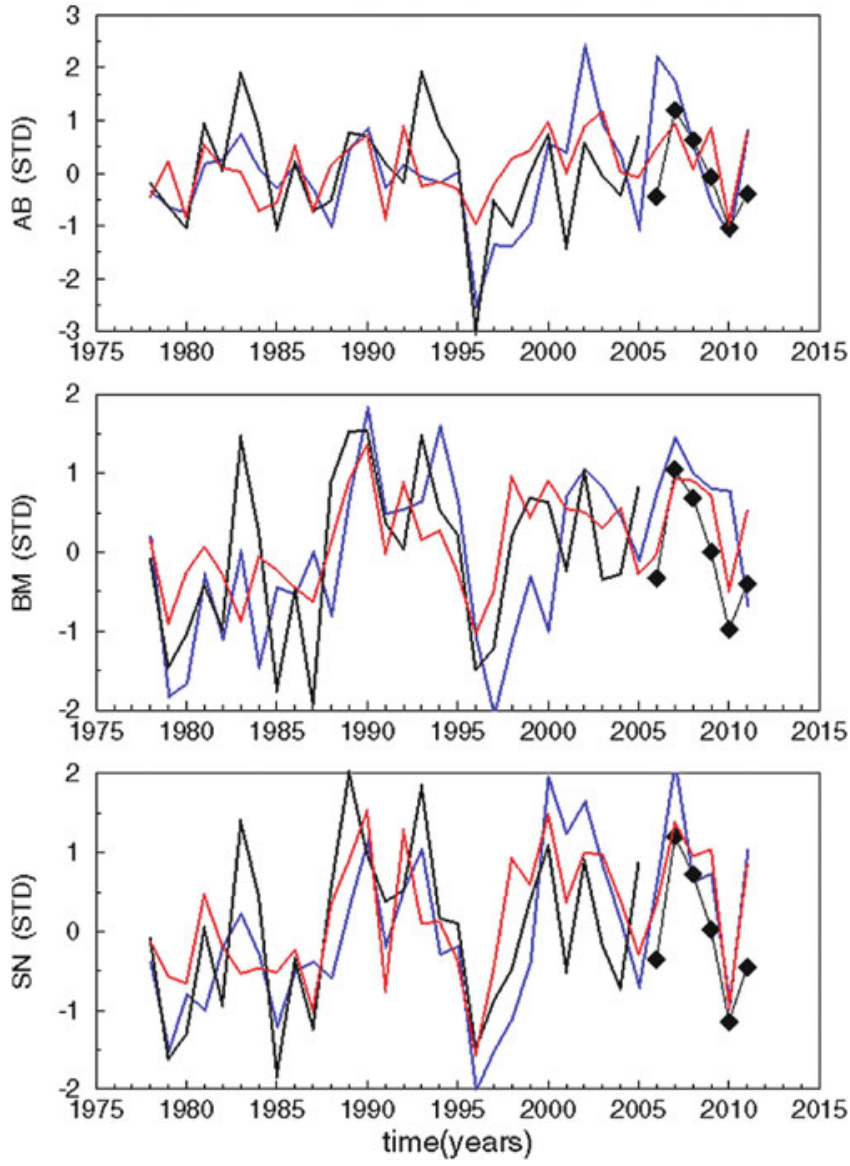

- Abb. 5.2 Vergleich von Modellergebnissen und Felddaten. Modellierte Datenreihe (Winter-NSE und Infauna 2. Quartal (blaue Linie) und 2. Quartal NSE und 2. Quartal Infauna (rote Linie) aus standardisierten Anomalien der log Abundanz (AB), log Biomasse (BM) und Artenzahl (SN) der InfaunaGemeinschaften vor Norderney, Felddaten der „Fitting"-Periode 1978-2005 (schwarze Linie) und unabhängigen Felddaten in der 6-Jahres-Vorhersageperiode 2006-2011 (Linie mit Diamant)). (Aus Dippner und Kröncke 2015)

Regimeshift, der zu höheren Varianzen in der Abundanz, der Artenzahl und der Biomasse der Infauna geführt hat. Um bessere Vorhersagen treffen zu können, haben Dippner und Kröncke (2015) einen neuen Klimaprädiktor für die südliche Nordsee entwickelt, den North Sea Environment (NSE) Index, in den die Arktische Oszillation, die Oberflächentemperatur der südlichen Nordsee, der Niederschlag sowie zonale und meridionale Winde einfließen. Die Korrelationen zwischen der Infauna und dem NSE-Index sind nach 2001 wieder signifikant (• Abb. 5.2). Das weist darauf hin, dass großräumige Veränderungen im Klimasystem auf der nördlichen Hemisphäre die Infauna-Gemeinschaften in der südlichen Nordsee beeinflussen.

Die Infauna-Gemeinschaften der Doggerbank wurden 2006/2007 beprobt und die Daten mit eigenen Daten aus den 1980er-, 1990er- und 2000er-Jahren und weiteren Daten aus den 1950er- und 1920er-Jahren verglichen (Kröncke 2011). Der Langzeitvergleich zeigte, dass zu Beginn des 20. Jahrhunderts die Intensivierung der Fischerei auf der Doggerbank zu einer Dezimierung von großen Muschelbänken führte, die sich bis heute aufgrund der kontinuierlichen Fischerei nicht erholt haben. Seit 1985 werden zudem durch die Temperaturerhöhung bedingte Veränderungen in den Infauna- und Epifauna-Gemeinschaften

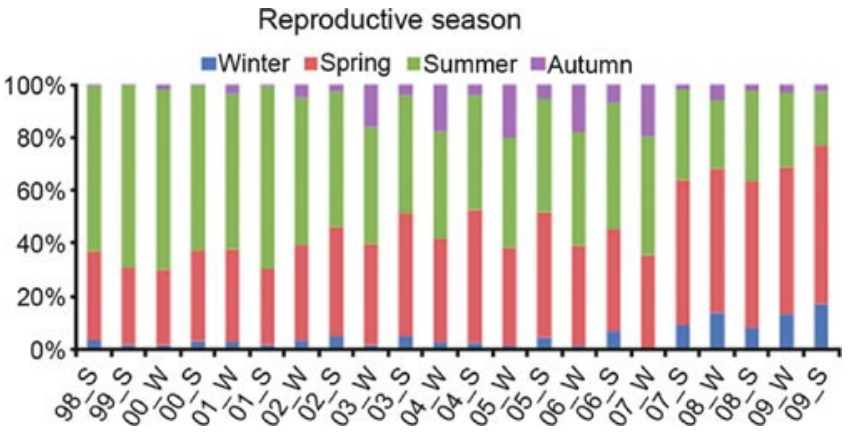

- Abb. 5.3 Veränderung in der Reproduktionszeit der Epifauna in Verbindung mit der Erhöhung der Oberflächentemperatur der Deutschen Bucht von 1998 bis 2009. (Aus Neumann und Kröncke 2011)

der Doggerbank beobachtet, die zu einer Zunahme von heimischen wärmeliebenden und einer Abnahme von kälteliebenden Arten geführt haben (Kröncke 2011).

Ein Beispiel für das Auftreten von neuen Arten in der Nordsee ist die Trapezkrabbe Goneplax rhomboides. Habitatmodelle deuten darauf hin, dass Wassertemperaturen von mehr als $5,5^{\circ} \mathrm{C}$ im Winter ihre Etablierung in der Nordsee begünstigt haben. Tatsächlich gab es seit 1996/1997 keinen extrem kalten Winter mehr, und auch die mittlere Wassertemperatur für die kalten Monate Februar und März war von 1997 bis 2010 mit $6{ }^{\circ} \mathrm{C}$ überdurchschnittlich hoch (Neumann et al. 2013a).

Die ökologische Funktion von Benthos-Gemeinschaften lässt sich besser durch ihre funktionelle Zusammensetzung beschreiben als durch die Artenzusammensetzung per se. Veränderungen in der funktionellen Zusammensetzung von EpifaunaGemeinschaften wurden in der Deutschen Bucht zwischen 1998 und 2009 anhand von 12 funktionellen Eigenschaften untersucht (Neumann und Kröncke 2011). Seit 2002 kam es zu einer Abnahme von Eigenschaften der Arten, die charakteristisch für eine angepasste Lebensweise sind. Diese traten vermehrt im Zusammenhang mit dem kalten Winter 1995/96 auf, in dessen Folge es zu einer Sukzession nach dem temperaturbedingten Massensterben der benthischen Fauna kam.

Mit den steigenden Oberflächentemperaturen seit 2001 korrelierten die Zunahme von Reproduktionszeiten im Herbst und Winter (• Abb. 5.3). Die Ergebnisse deuten daher darauf hin, dass sich Klimaveränderungen primär auf die Reproduktion der Epifauna und weniger auf ihre Ernährungstypen durch ein verändertes Nahrungsangebot auswirken.

\subsubsection{Fische}

Nach wie vor bestimmt die Fischerei die Fischbestände der Nordsee am stärksten. Als Reaktion auf zurückgehende Fischbestände wurden die Regularien der gemeinsamen Fischereipolitik der EU verschärft und schränken die Fischerei in den letzten Jahren zunehmend ein (EAA 2015). Des Weiteren wird die Fischerei in Zukunft durch die zunehmenden Installationen zur Gewinnung von Offshore-Windenergie wie auch durch die Einrichtung von Schutzgebieten aus manchen Gebieten fast vollständig verdrängt werden (Berkenhagen et al. 2010). Es ist daher schwierig, die Ein- 
flüsse des sich ändernden Klimas auf die Fischbestände im Wechselspiel mit weiteren anthropogenen Einflüssen zu bewerten.

Ein weiteres Problem bei der Bewertung der sich ändernden Zustände ist die relative Kürze bestehender Zeitserien systematischer Aufnahmen der Nordsee-Fischfauna. Oft reichen diese bestenfalls nur einige Dekaden zurück, wie z. B. die Aufnahmen des International Bottom Trawl Surveys, die konsistente Abundanzindizes für Nordseefische längstens bis in die späten 1970er-Jahre liefern (Heessen et al. 2015). Damit überspannen diese Beobachtungen nur wenige Zyklen mittelfristiger Klimaschwankungen wie die der Nordatlantischen Oszillation (NAO) und kaum einen Zyklus der langfristigen Multidekadischen Atlantischen Oszillation (AMO). Die Bedeutung dieser Zyklen für die Fischfauna der Nordsee wurde mehrfach nachgewiesen und reicht aus, die meisten zurzeit zu beobachtenden Änderungen in der Fischfauna zu erklären (z. B. Gröger et al. 2010; Alheit und Bakun 2010). Auch die im 1. HKB (Colijn und Fanger 2011) beschriebenen Änderungen in der Fischfauna - insbesondere das Auftreten lusitanischer Arten in der südlichen Nordsee - können mit den natürlichen Wechseln kalter und warmer Phasen des Klimas erklärt werden. Gerade die beiden Arten Roter Knurrhahn (Chelidonichthys lucerna) und Streifenbarbe (Mullus surmuletus) sind keine neuen „Player“ im Nordseeökosystem (vgl. Heincke 1894). Das verstärkte Auftreten des Roten Knurrhahns insbesondere in der Mitte der 1990er-Jahre (Sell und Heessen 2015) scheint eher im Zusammenhang mit dem gut dokumentierten Regimewechsel Ende der 1980er-Jahre (z. B. Schlüter et al. 2008) zu stehen.

Genauso kann der beschriebene Wechsel von einer Gadidendominierten zu einer Plattfischdominierten Fischgemeinschaft in der deutschen AWZ eher als die Rückkehr zum Normalzustand nach dem Gadoid-Outburst in den späten 1960er- bis frühen 1980er-Jahren gesehen werden denn als eine Folge des Klimawandels (vgl. Hislop et al. 2015, S. 193). Der Gadoid-Outburst beschreibt eine Phase starker Zunahmen in den kabeljauartigen Beständen der Nordsee, deren Aufblühen und Niedergang oft mit langfristigen Schwankungen des Klimas in Verbindung gebracht wird (Beaugrand 2009). Jedoch zeigen neuere Analysen, dass ohne den Einfluss der Fischerei sowohl der starke Anstieg als auch das Zusammenbrechen der Bestände nur schwer zu erklären sind (Fauchald 2010). Dieses Beispiel unterstreicht deutlich die Verwobenheit klimatischer und anthropogener (hier Überfischung) Einflüsse auf Ökosysteme. Diese aufzulösen und anthropogene von klimatischen Einflüssen zu trennen ist schwierig, aber unerlässlich, um realistische Vorhersagen für die Folgen des Klimawandels auf das Nordsee-Ökosystem und seine Fischbestände zu tätigen (Möllmann und Diekmann 2012).

Rijnsdorp et al. (2010) formulieren eine Reihe von Arbeitshypothesen zu möglichen Auswirkungen des Klimawandels auf die Physiologie und die Ökologie der Fische, durch deren Prüfung eine Abschätzung zu den Auswirkungen des Klimawandels auf Fischbestände ermöglicht werden soll. Diese werden im Folgenden stark verkürzt wiedergegeben.

1. Populationen an den Verbreitungsgrenzen ihrer Art werden stärker beeinflusst als solche, die sich im Zentrum der Verbreitung befinden. So können z. B. die erhöhten Temperaturen im Sommer den Sauerstoffhaushalt der eher kältelieben- den Arten negativ beeinflussen und deren Verschwinden aus der Nordsee begünstigen (Pörtner und Knust 2007).

2. Habitat- und Nahrungsansprüche der Arten spielen eine übergeordnete Rolle. So werden pelagische Fische anders beeinträchtigt als demersale Arten, da Erstere in Bezug auf Änderungen im Wasser anders reagieren können als Letztere, deren Habitate geographisch stärker eingeschränkt sind.

3. Fischarten mit räumlich stark eingeschränkten Habitatansprüchen während mindestens eines Teils ihres Lebenszyklus reagieren empfindlicher auf den Klimawandel als solche ohne spezifische Ansprüche. Dies gilt insbesondere für die frühen Lebensstadien von Fischen, die oft ein engeres Temperaturoptimum haben als ältere Stadien (Rijnsdorp et al. 2010).

4. Fischbestände reagieren unter dem Einfluss des Klimawandels empfindlicher auf Fischereidruck. Neben der Dezimierung des Laicherbestandes übt Fischerei durch den Wegfang großer Tiere einen Selektionsdruck auf Fischpopulationen aus. So werden z. B. Individuen, die früher und bei kleinerer Größe zur Geschlechtsreife gelangen, bevorzugt (Rijnsdorp et al. 2010), was den Genpool verändert. Dies kann negative Auswirkungen auf die Möglichkeiten der Anpassung an Umweltveränderungen haben.

Der Klimawandel könnte sich zukünftig auch indirekt auf die Fischgemeinschaft der Nordsee auswirken, indem der Mensch auf den Klimawandel reagiert (EEA 2015). Durch die vermehrte Gewinnung erneuerbarer Energie aus Offshore-Windparks werden in großem Umfang künstliche Hartsubstrate in die Nordsee eingebracht und damit Habitate für Arten geschaffen, die sonst nicht in den betreffenden Gebieten vorkommen (Ehrich et al. 2007). Auf der anderen Seite werden dadurch auch Schutzgebiete für Fische eingerichtet, da Fischerei in diesen Gebieten weitgehend untersagt sein wird.

Der Klimawandel im Zusammenspiel mit den anthropogenen Einflüssen wird Auswirkungen auf die Fischbestände und die Fischerei der Nordsee haben (Hollowed et al. 2013). Es besteht nach wie vor immenser Forschungsbedarf auf Basis der oben aufgezeigten Arbeitshypothesen, um die Folgen realistisch abschätzen zu können (Rijnsdorp et al. 2009, 2010; Hollowed et al. 2013).

\subsection{Wattenmeer}

Das Wattenmeer ist das größte zusammenhängende intertidale Gezeitengebiet der gemäßigten Breiten und wurde 2009 als Weltnaturerbe anerkannt. Begründet wurde dies mit der jungen und ursprünglichen, durch Wind und Gezeiten ständig neu geformten Landschaft, in der Naturkräfte walten und sich eine enorme Vielfalt des Lebens entwickeln kann. Die bestehenden Regelungen garantieren die Wahrung dieses Gebietes. Fortschritte wurden bei der Reduzierung von Kontaminanten und Nährstoffen erzielt. Problematisch sind aber weiterhin die hohe Anzahl eingeschleppter nichtheimischer Arten, und unsicher ist, ob der Aufwuchs der Gezeitenflächen und Salzwiesen mit dem steigenden Meeresspiegel (s. auch $>$ Abschn. 4.1) Schritt halten kann (Wolff et al. 2010; Reise et al. 2010). 


\subsubsection{Plankton}

Die für die Nordsee beschriebenen allgemeinen Effekte einer Klimaänderung auf das Plankton, wie eine Tendenz zu kleineren Arten, ein potenzieller Mismatch zwischen Phyto- und Zooplankton und eine höhere Produktivität, treffen im Prinzip auch auf das Wattenmeer zu (vgl. Winder und Sommer 2012). Wie die tatsächliche Auswirkung genau sein wird, ist unklar (Philippart und Epping 2009) und wird von lokalen Gegebenheiten abhängen (vgl. Cloern und Jassby 2010). Insbesondere können Wechselwirkungen mit dem filtrierenden Makrozoobenthos eine entscheidende Rolle spielen: Sowohl eine Zunahme der FilterFeeder z. B. durch eingewanderte Arten (s. u.) als auch eine Abnahme infolge wärmerer Winter (schlechtere Rekrutierung) sind möglich (Philippart und Epping 2009).

Während saisonale und interannuelle Dynamik des Phytoplanktons der offenen Nordsee stark durch Temperatur und Hydrographie bestimmt werden, spielen im Wattenmeer und im angrenzenden Küstenwasser Nährstoffe eine zusätzliche Rolle. Infolge der Abnahme der Nährstofffrachten durch Rhein, Maas, Weser, Ems und Elbe sind Algenblüten und Biomasse im Wattenmeer vor allem im Sommer zurückgegangen (Cadée und Hegeman 2002; Philippart et al. 2007; van Beusekom et al. 2009a, 2009b). Einige Wissenschaftler erwarten, dass die Abflüsse im Sommer ab- und im Winter zunehmen (z. B. Huang et al. 2010) und dass die Nährstofffrachten im Sommer ab- und im Winter zunehmen werden (z. B. Andersen et al. 2006). Aber auch gegenläufige Szenarien mit zunehmenden Abflüssen und Nährstofffrachten im Sommer und abnehmenden Abflüssen und Nährstofffrachten im Winter sind möglich ( Abschn. 4.1 und 4.2). Die Nährstoffumsätze werden mit steigenden Temperaturen wahrscheinlich ansteigen (Beck und Brumsack 2012) und in Kombination mit höheren Temperaturen die Produktivität erhöhen. Beide Faktoren werden verstärkt zu anoxischen Bedingungen in Sedimenten und demzufolge zu verstärkten Phosphorfluxen führen. Da das Wattenmeer zumindest im Frühjahr phosphat-limitiert sein kann (Ly et al. 2014), wird eine erhöhte Temperatur die Frühjahrsblüte im Prinzip stärken.

Ein Temperaturanstieg kann das Potenzial von Frühjahrsblüten prinzipiell erhöhen. Aber auch die Top-down-Kontrolle von Phytoplankton wird insbesondere durch höhere Wintertemperaturen nachweislich gestärkt: Langzeitdaten aus dem nordfriesischen Wattenmeer bei List/Sylt zeigen, dass der Umfang der Frühjahrsblüte mit zunehmender Temperatur abnimmt (van Beusekom et al. 2009b; - Abb. 5.4). Auch das Zooplankton reagiert stark auf die Wintertemperaturen (Martens und van Beusekom 2008) mit einer früheren Entwicklung des Zooplanktons und höheren mittleren Biomassen nach warmen Wintern. Ein Treiber für die geringere und später einsetzende Frühjahrsblüte scheint das verstärkte Copepodengrazing in und nach warmen Wintern zu sein (s. auch Wiltshire und Boersma 2016). Auch eine temperaturabhängige Kontrolle durch benthische Filter-Feeder kann zum erhöhten Grazingdruck beitragen (Keller et al. 1999). Weil Wachstums- und Grazingpotenzial zunehmen, wird sich die Dynamik mit ungewissem Endergebnis erhöhen.

Neben dem Phytoplankton trägt auch das Phytobenthos zur Produktivität des Wattenmeeres bei. De Jonge et al. (2012) be-

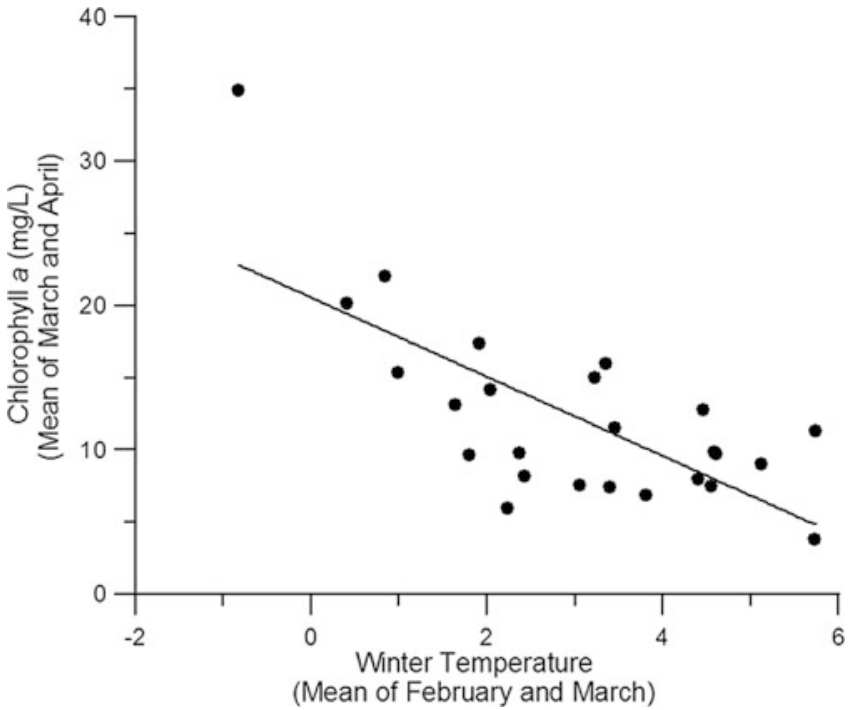

- Abb. 5.4 Die Auswirkung der Wintertemperatur (Mittelwert Februar bis März; 1980-2007) auf die mittlere Phytoplanktonbiomasse (als Chlorophyll a) der Frühjahrsblüte (Mittelwert März bis April). (Aus van Beusekom et al. 2009b)

schrieben, dass eine höhere mittlere Jahrestemperatur zu einer erhöhten Phytobenthosbiomasse führt. Dieser Effekt ist nur z. T. auf physiologische Prozesse zurückzuführen und wird wahrscheinlich auch durch Top-down-Effekte hervorgerufen.

Die Reaktion des Wattenmeerplanktons auf den Klimawandel wird komplex sein und auch davon abhängen, wie sich Umweltpolitik und Klimaänderung auf die Nährstoffflüsse auswirken werden.

\subsubsection{Makrozoobenthos}

Der Klimawandel bedingt langfristig einen Wechsel der Dominanzverhältnisse von Organismen im Wattenmeer (s. 1. HKB; Colijn und Fanger 2011). Neben diesen gleitenden Entwicklungen sind es derzeit aber auch spontane Ereignisse und deren Folgen, welche die Sedimentlebensgemeinschaften der südöstlichen Nordseeküste grundlegend neu strukturieren. Globaler Schiffsverkehr und Aquakultur führen zu einem steten Einstrom von Organismen ferner Küstenlebensräume. Bis zum Jahr 2010 wurden 66 exotische Arten durch menschliches Handeln vor allem aus wärmeren Meeresgebieten eingeschleppt (Buschbaum et al. 2012). Eine seit 2009 durchgeführte Erfassung exotischer Arten zeigt, dass sich derzeit im Mittel etwa 1,5 bodenlebende Arten pro Jahr neu und dauerhaft etablieren, dreimal mehr als in der ersten Hälfte des 20. Jahrhunderts. Mit einem weiterhin anhaltenden Trend der Erwärmung finden die Neuankömmlinge geeignete Bedingungen vor, den Sprung ins Wattenmeer zu schaffen.

Es sind aber nicht nur die Neueinschleppungen, welche die Gemeinschaftsstrukturen des Wattenmeeres klimabedingt verändern. Besonders anschaulich wird dies bei der Australischen Seepocke Austrominius modestus ( $\bullet$ Abb. 5.5). Diese festsitzende und aus dem Pazifikraum stammende Krebsart ist schon zu Beginn des Zweiten Weltkrieges mit Kriegsschiffen in die Nordsee 

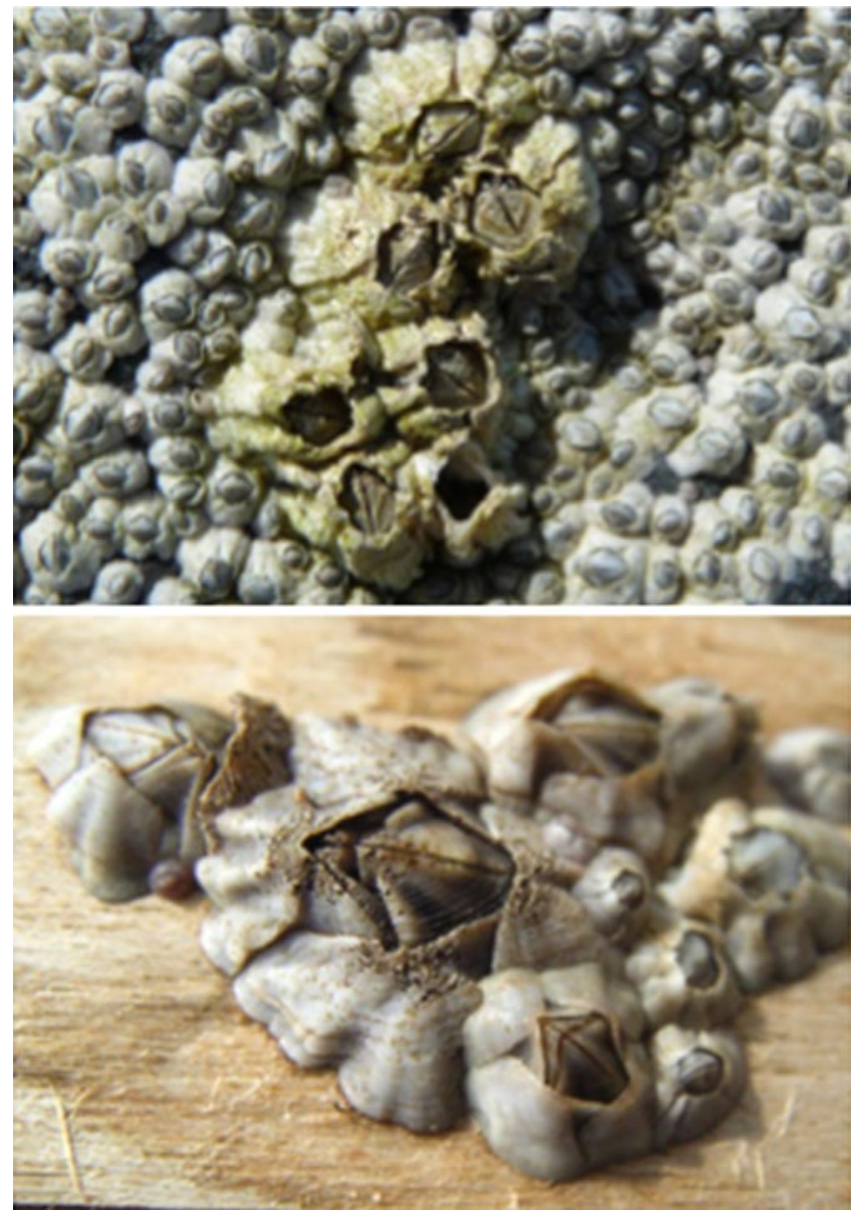

- Abb. 5.5 Wärmere Temperaturen führen zu Dominanzverschiebungen von Arten im Wattenmeer. Die boreal-arktische Seepocke Semibalanus balanoides (oben) leidet unter der Erwärmung, wobei die eingeschleppte Australische Seepocke Austrominius modestus (unten) zunimmt

eingebracht worden (Lackschewitz et al. 2015). Kalte Winter dezimierten immer wieder ihre geringe Populationsstärke. Eine Reihe warmer Sommer und milder Winter bewirkten eine exponentielle Zunahme des Vorkommens von A. modestus (Witte et al. 2010). Mitte der 1990er-Jahre lagen ihre Dichten bei wenigen Tieren pro $\mathrm{m}^{2}$, um bis 2008 auf etwa 100.000 Individuen pro $\mathrm{m}^{2}$ anzusteigen. Ähnliche Muster zeigen eine Reihe weiterer thermophiler eingeschleppter Arten, die von den ansteigenden Temperaturen profitieren und mit einem anschließenden Massenvorkommen reagieren. Dazu gehören die Pazifische Auster Crassostrea gigas und auch die Amerikanische Pantoffelschnecke Crepidula fornicata (Buschbaum und Reise 2010).

Im Jadebusen, einer Bucht im Nationalpark Niedersächsisches Wattenmeer, lassen sich die Veränderungen der InfaunaGemeinschaften über einen Zeitraum von fast acht Jahrzehnten überblicken (Schückel und Kröncke 2013; Schückel et al. 2013). Die Artenzahl stieg zwischen 1930, 1970 und 2009 teils durch eingeschleppte Arten an. Die seit 1997 vorherrschenden milden Winter führten zu einem starken Abundanzrückgang der heimischen Muschelpopulationen (Mytilus edulis, Macoma balthica, Cerastoderma edule). Abundanzen ehemals charakteristischer Arten wie des Schlickwattkrebses Corophium volutator (Schlickwatt), des Flohkrebses Bathyporeia sarsi (Sandwatt) oder des
Vielborstenwurms Pygospio elegans (Mischwatt) nahmen ebenfalls ab. Eine starke Zunahme zeigte dagegen der Wenigborstenwurm Tubificoides benedii (Schlickwatt) zwischen 1930 und 2009. Mögliche Ursachen könnten Veränderungen in der Sedimentzusammensetzung und der Morphologie der Watten sein, die durch den Meeresspiegelanstieg bedingt sind.

Ein Vergleich der benthischen Nahrungsnetze im Jadebusen mithilfe der „Ecological Network Analysis“ (ENA) zeigte, dass Eutrophierungseffekte zwischen den 1930er- und 1970er-Jahren dazu geführt haben, dass weniger organisches Material im benthischen System wiederverwendet wurde und der Energietransfer des benthischen Nahrungsnetzes abnahm, während er zwischen den 1970er-Jahren und 2009 in Verbindung mit Temperaturerhöhung und warmen Wintern und einem höheren Sedimenteintrag anstieg (Schückel et al. 2015).

Insgesamt verursacht der Klimawandel weitreichende Folgen im Wattenmeer. Langfristig werden sich heimische kälteliebende Arten in höhere Breiten zurückziehen, weitere Organismen aus südlicheren Gebieten ihr Verbreitungsareal nach Norden ausdehnen und eingeschleppte Arten von wärmeren Küsten sich weiterhin etablieren.

\subsubsection{Fische}

Nur ein geringer Anteil der Fischarten des Wattenmeeres vollzieht den gesamten Lebenszyklus auch dort. Vielmehr prägen wandernde Arten die Fischfauna des Wattenmeeres, weswegen die Mechanismen ihrer strukturellen und funktionellen Veränderungen infolge oder unter Beteiligung des Klimawandels grundsätzlich denen der Nordsee und den Flüssen entsprechen oder dort oft sogar ihre Ursachen haben.

Selten ist ausschließlich der Klimawandel für tief greifende Veränderungen der Fischgemeinschaften verantwortlich. Als wiederkehrendes Muster manifestiert die Kombination von Überfischung und Klimaveränderungen einen alternativen Ökosystemzustand in der Nordsee (Kirby et al. 2009), der mitunter bis ins Wattenmeer wirkt.

Aktuelle Analysen der Abundanzmuster von Fischen im Niederländischen Wattenmeer zwischen 1960 und 2011 ergaben einen signifikanten Zusammenhang mit dem NAO-Winterindex und der Chlorophyll-a-Konzentration im Sommer. Dieser Zusammenhang war bei verwandten Fischarten mit vergleichbaren Lebenszyklen sehr ähnlich (van der Veer et al. 2015). Es konnte eine starke Abnahme der kaltadaptierten Aalmutter Zoarces viviparus (vgl. Pörtner und Knust 2007) bei gleichzeitiger Zunahme des warmadaptierten Wolfsbarsches Dicentrarchus labrax (vgl. Henderson und Corps 1997) nachgewiesen werden. Diese auch im Wattenmeer beobachteten Trends werden einer klimabedingten Verschiebung der Verbreitungsgebiete dieser beiden Arten mit spezifischen Temperaturpräferenzen und -toleranzen zugeschrieben und entsprechen nordseeweiten Beobachtungen (Perry et al. 2005).

Seegraswiesen des Eulitorals weisen nicht nur höhere Dichten an mobilem Epibenthos auf als andere Habitate (Polte et al. 2005). Sie bieten auch saisonal massenhaft auftretenden Fischarten wie Dreistachligen Stichlingen Gasterosteus aculeatus, 
Heringen Clupea harengus und Hornhechten Belone belone ein wichtiges Laichhabitat (Polte und Asmus 2006a, 2006b), wodurch eine Veränderung dieses Habitattyps im Wattenmeer auch den Lebenszyklus der o. g. Fischarten beträfe. Während sich die eulitoralen Seegrasbestände insbesondere des schleswig-holsteinischen Wattenmeeres jüngst positiv entwickelten und eine direkte Auswirkung höherer Wassertemperaturen als unwahrscheinlich erachtet wird (van der Graaf et al. 2009), sind die Seegraswiesen sensibel gegenüber instabilen Sedimenten (Cabaco und Santos 2007). Dieser Umstand könnte sich in der Folge von häufigeren und stärkeren Sturmereignissen indirekt auf Qualität und Quantität dieses Fischhabitats auswirken.

Ein weiteres Habitat, das grundsätzlich von Fischen genutzt wird, sind tidebeeinflusste Salzmarschen (Mathieson et al. 2000). Mit über 40.000 ha beherbergt die Wattenmeerregion etwa $20 \%$ aller europäischen Salzmarschenflächen (Doody 2008), deren Priele und Grüppen bei entsprechenden Wasserständen von einer Vielzahl an Fischarten genutzt werden. Küstenmarschen gehören zu den potenziell am stärksten von einem steigenden Meeresspiegel betroffenen Habitaten (Kirwan und Megonigal 2013).

Eine der auffälligsten strukturellen Veränderungen der Fischfauna des Wattenmeeres ist das vermehrte Auftreten der Sardelle Engraulis encrasicolus. Dieser warmadaptierte pelagische Schwarmfisch reproduziert nach ca. 40 Jahren weitgehender Abwesenheit aus Nordsee und Wattenmeer seit Mitte der 1990erJahre wieder erfolgreich im Gebiet (Boddeke und Vingerhoed 1996). Allerdings wurden im Wattenmeer seit Anfang des 20. Jahrhunderts wiederholt Laichereignisse beschrieben (Meyer 1930; Aurich 1953), sodass die Laichereignisse der jüngeren Vergangenheit möglicherweise nur eine weitere Beschreibung eines dauerhaften Laichvorkommens variabler Intensität und Ausdehnung sind.

\subsection{Elbeästuar}

Flussmündungen (Ästuare) wie die Tideelbe sind Übergangszonen, in denen Süßwasser- und Meereslebensräume aufeinandertreffen. Obwohl weltweit nur 5,8 \% aller Küstengebiete Ästuare sind (Wetzel et al. 2014), sind sie von hohem ökologischem Wert für viele Pflanzen- und Tierarten (Barbier et al. 2011). Gleichzeitig sind viele Ästuare weltweit stark vom Menschen beeinflusst (Ahlhorn 2009; s. auch $\longrightarrow$ Abschn. 4.2). Sie gehören heute mit zu den am stärksten anthropogen überformten aquatischen Lebensräumen auf der Erde: durch Deichbau und Landgewinnung (Barbier et al. 2011), Befestigung der Ufer mit Steinschüttungen (Wetzel et al. 2014), Vertiefungen von Fahrrinnen, ihre Unterhaltung (z. B. Drabble 2012) und die Verbringung des Sediments (z. B. Taupp und Wetzel 2013) sowie durch die Belastung mit chemischen Substanzen (z. B. Wetzel et al. 2013).

\subsubsection{Plankton}

Die Produktivität des Phytoplanktons im Elbeästuar ist stark lichtlimitiert (Fast und Kies 1990). Nach Winder und Sommer (2012) ist daher zu erwarten, dass wärmere Temperaturen kaum zu einer höheren Produktivität führen werden, die heterotrophen Prozesse aber deutlich gestärkt werden. Schon heute wird die meiste Organik nicht lokal produziert, sondern in das Ästuar importiert. Quellen sind sowohl das Meer als auch die Elbe (van Beusekom und Brockmann 1998). Diese Quellen haben sich in den letzten Dekaden deutlich geändert. Die Produktivität des Küstenwassers ist infolge der Abnahme der Nährstoffeinträge zurückgegangen (Philippart et al. 2007; van Beusekom et al. 2009a). Dementsprechend kann angenommen werden, dass auch der Eintrag von partikulärem organischem Material aus der Nordsee abgenommen hat. Die Frachten von organischem Material aus der Elbe haben sich aber deutlich geändert: Amann et al. (2012) beschrieben eine Zunahme der Phytoplanktonblüten in der Elbe trotz abnehmender Nährstofffrachten infolge einer verringerten Toxizität des Elbewassers. Die Algenblüten entwickeln sich im Oberlauf der Elbe (Scharfe et al. 2009), können sich aber im limnischen Teil des Elbeästuars infolge der schlechten Lichtbedingungen nicht weiter entwickeln, sterben entweder durch Lichtlimitierung oder durch Grazing ab und führen letzten Endes zu sehr niedrigen Sauerstoffkonzentrationen (Schöl et al. 2014). Es ist zu erwarten, dass ein Temperaturanstieg sowohl die Blütenbildung in der Elbe als auch das Sauerstoffdefizit verstärken wird. Die Rolle des Zooplanktons und die Folgen für das Zooplankton sind wegen mangelnder Forschung unklar.

Flussvertiefungen können die Schwebstoffkonzentrationen des Ästuars stark erhöhen, wie z. B. in der Ems geschehen (de Jonge et al. 2014). Obwohl an sich nicht klimarelevant, wären schlechtere Lichtbedingungen und weniger Sauerstoffproduktion zu erwarten. Die Folgen eines Klimawandels würden die Ausbildung von Sauerstoffdefiziten verstärken. Für das Zooplankton haben höhere Schwebstoffkonzentrationen wahrscheinlich eine geringere Fruchtbarkeit zur Folge (Gasparini et al. 1999). Es ist wahrscheinlich, dass diese Art von interaktiven Effekten die zukünftige Entwicklung des Planktons prägen wird.

Wie im letzten Klimabericht schon festgestellt, fehlen rezente Forschungsergebnisse, um eine fundierte Aussage über die Änderung des Planktonsystems im Elbeästuar bzgl. Klimaänderung machen zu können. Es gibt zwar eine Reihe von Beobachtungen aus anderen Ästuaren, die Hinweise auf mögliche Änderungen geben. Es ist aber festzuhalten, dass ästuarine Systeme schwer zu vergleichen sind, weil die Phänologie der Planktondynamik stark von lokalen Effekten geprägt wird (z. B. Cloern und Jassby 2010).

\subsubsection{Makrozoobenthos}

Die Makrobenthosfauna von Ästuaren ist generell weit weniger arten- und individuenreich als die Mikro- und Meiofauna, weist aber eine deutlich höhere Biomasse auf (Kennish 2002). In der Tideelbe ist die mittlere Abundanz des Makrozoobenthos im Bereich des Trübungsmaximums (etwa bei Flusskilometer 690) mit 4414 Individuen $/ \mathrm{m}^{2}$ am höchsten, während die mittlere Artenzahl im äußeren Bereich (Flusskilometer 730-740) mit 5,1 Arten am höchsten ist (Taupp und Wetzel 2014).

Das Makrozoobenthos in der Tideelbe ist einer Vielzahl von anthropogenen Stressoren ausgesetzt, die einen Einfluss auf die Verbreitung der einzelnen Arten haben. Die chemische 
Belastung des Sediments (Wetzel et al. 2013) spielt - neben der rein mechanischen Störung durch z. B. Umlagerung von Sedimenten (Taupp und Wetzel 2013) - eine wichtige Rolle für die Benthosfauna und den ökologischen Zustand des Gewässers (Wetzel et al. 2012). Diese Stressoren sorgen dafür, dass die Individuenzahlen der meisten Benthosarten geringer als möglich ausfallen, obwohl Nahrung ausreichend vorhanden ist. Alle diese anthropogenen Stressoren beeinflussen die Benthosfauna und erschweren das Auffinden klimarelevanter Veränderungen in Ästuaren. Weltweit sind solche Untersuchungen in Ästuaren bisher sehr selten und beschränken sich meist auf die Diskussion potenzieller Einflüsse des Klimawandels (Struyf et al. 2004) oder auf spezielle Themen wie z. B. die benthisch-pelagische Kopplung (Fulweiler und Nixon 2009). Das Hauptproblem ist das Fehlen historischer Langzeitdaten (Bianchi 1997). Für das Elbeästuar stehen derzeit Daten für das Makrozoobenthos nur seit 1997 aus dem Ästuarmonitoring der Bundesanstalt für Gewässerkunde zur Verfügung (Nehring und Leuchs 1999). Ein Vergleich mit historischen Daten für die Tideelbe seit 1954 (- Abb. 5.6) zeigt vor allem starke Schwankungen in der Abundanz des Makrozoobenthos, dem Vorkommen aller Individuen, und der Anzahl der Arten. Dies ist nicht ungewöhnlich und in Ästuaren häufig zu beobachten (McLusky et al. 1993). Ein klarer statistisch signifikanter Trend ist hier nicht auszumachen und noch weniger einem einzelnen Einflussfaktor wie dem Klimawandel zuzuordnen. Selbst das extreme Hochwasser der Elbe im Jahr 2002 ist in diesen Daten nicht eindeutig identifizierbar. Gerade solche Extremereignisse sollen aber laut den Projektionen über den Klimawandel in den nächsten Jahrzenten zunehmen (Bronstert 1995; Menzel und Bürger 2002). Untersucht man die zeitliche Veränderung des Makrozoobenthos auf Artniveau, so werden die Schwankungen zwischen den einzelnen Jahren noch deutlicher: An der Station Flusskilometer 749 (vgl. • Abb. 5.6) wechseln von einem zum anderen Jahr bis zu $60 \%$ der Arten. Solche Schwankungen machen eine genaue Identifikation möglicher klimabedingter Veränderungen derzeit unmöglich; die bisher vorhandene Datenbasis reicht noch nicht aus, um belastbare Aussagen über den Einfluss des Klimawandels auf das Makrozoobenthos in der Tideelbe zu machen.

Schon ein relativ kleiner Anstieg der Wassertemperatur kann im Prinzip aber zu einer deutlichen Verschiebung der Verbreitungsgrenzen von Arten führen (Brierley und Kingsford 2009). Wärmeliebende Arten würden sich so weiter ausbreiten, während kälteliebende Arten sich zurückziehen oder verschwinden würden. Gerade im Hinblick darauf, dass eingewanderte Arten (Neozoen) in der Regel wärmeliebend sind, ist anzunehmen, dass diese aufgrund ihrer physiologischen Fähigkeiten (Becker et al. 2015) in ihrer Verbreitung besonders bevorzugt sind (Orendt et al. 2010), sodass auch in der Tideelbe in Zukunft mit einem erhöhten Vorkommen von Neozoen zu rechnen ist (Nehring 2006).

Insgesamt kann davon ausgegangen werden, dass der Klimawandel auch im Elbeästuar zu einer deutlichen Veränderung der Makrobenthosfauna führen wird. Die Zunahme von extremen Abflussereignissen aufgrund von starken Regenfällen oder lang anhaltenden Trockenperioden bevorzugt Arten, die an stark wechselnde Lebensbedingungen angepasst sind. Langfristig ist auch mit einem erhöhten Vorkommen von wärmeliebenden

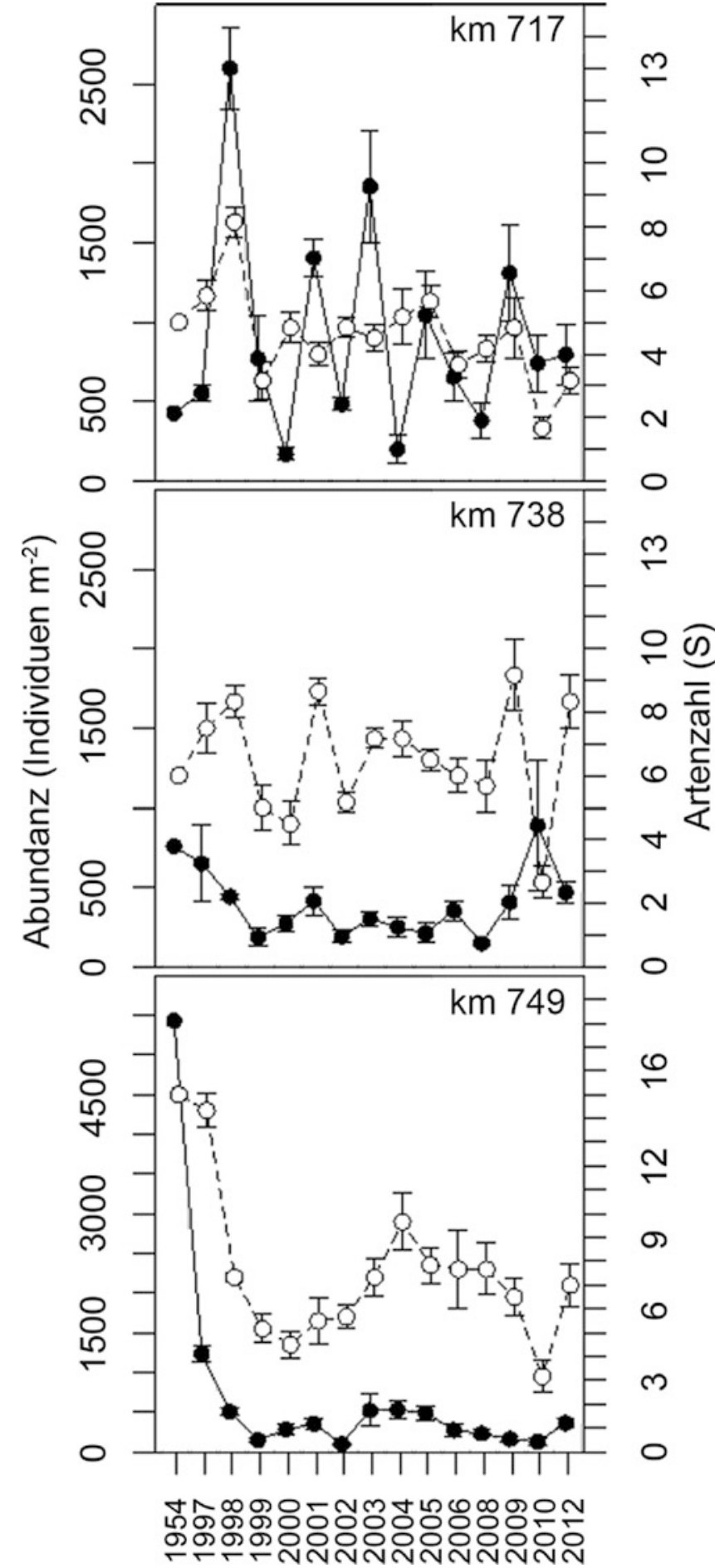

- Abb. 5.6 Verteilung der Makrobenthos-Abundanzen entlang des Elbeästuars

Neozoen zu rechnen. Um Untersuchungen zum Einfluss des Klimawandels auf die Benthosfauna im Elbeästuar voranzutreiben, muss die vorhandene Datenbasis weiter ausgebaut und, wo möglich, um Daten aus älteren Veröffentlichungen ergänzt werden. Zusammen mit den sich, gerade in letzter Zeit, immer weiter entwickelnden mathematischen Analyseverfahren sollten damit in Zukunft genauere Aussagen über klimabedingte Veränderung der Benthosfauna möglich sein. 


\subsubsection{Fische}

Im Elbeästuar zwischen Stade und Cuxhaven wurden im Zeitraum 1981-2010 insgesamt 64 Fischarten nachgewiesen (Möller 1984, 1988; Thiel et al. 1995; Thiel und Potter 2001; Eick und Thiel 2014). Die darunter befindlichen 49 marinen, ästuarinen und diadromen Arten lassen sich einer der drei biogeographischen Kategorien lusitanisch (südlich), boreal (nördlich) bzw. atlantisch zuordnen (z. B. Dulvy et al. 2008; Engelhard et al. 2011).

In der ersten Hälfte der 1990er-Jahre und im Zeitraum 20092010 war im Brackwasserbereich der Elbe eine höhere Anzahl lusitanischer Fischarten zu verzeichnen als in der ersten Hälfte der 1980er-Jahre (• Abb. 5.7), was jedoch nicht eindeutig auf einen Einfluss des Klimawandels zurückgeführt werden kann. Auch Einflüsse mittelfristiger Klimaschwankungen und/oder anthropogener Faktoren können hierfür Ursachen sein.

Bei Verwendung der gleichen Erfassungsmethodik mit kommerziellen Hamenkuttern auf jeweils vier in allen Untersuchungsperioden ähnlich lokalisierten Fangstationen wurden im Elbeästuar zwischen Stade und Cuxhaven von 1981 bis 1986 insgesamt 9, von 1990 bis 1994 dagegen 21 und von 2009 bis 2010 immerhin 16 lusitanische Fischarten (• Abb. 5.7) gefangen. Die Anzahl atlantischer und borealer Fischarten blieb hier seit den 1980er-Jahren mit 2-3 bzw. 15-19 Arten dagegen relativ konstant.

Innerhalb der Gruppe der schon in den 1980er-Jahren nachgewiesenen und aktuell mit Präsenzen von über $10 \%$ vorkommenden heimischen lusitanischen Arten ist ein stetiger Anstieg der Präsenzen seit den 1980er-Jahren bei der Finte (Alosa fallax) und der Kleinen Seenadel (Syngnathus rostellatus) festzustellen (- Abb. 5.7). Für den Laicherbestand der Finte im Elbeästuar ermittelten Magath und Thiel (2013) im Zeitraum 2009-2010 signifikant höhere Abundanzen im Vergleich zum Zeitraum 1992-1993 bei auch höheren mittleren Wassertemperaturen zu Beginn der Laichperiode der Art. Ein direkter Zusammenhang zwischen dem Rekrutierungserfolg bzw. der Laicherbestandsgröße der Finte und der Wassertemperatur wurde bei anderen Studien vermutet (z. B. Aprahamian et al. 2010; Thiel et al. 2008).

Auch der Etablierungserfolg der invasiven Schwarzmundgrundel (Neogobius melanostomus), die seit 2008 nachweislich im Hamburger Abschnitt der limnischen Tideelbe vorkommt (Hempel und Thiel 2013) und seitdem in ihrem Bestand anwächst (Thiel und Thiel 2015), scheint durch höhere Temperaturen begünstigt zu werden.

In Zukunft könnten höhere Wassertemperaturen in der Winterperiode in der Elbe den Fortpflanzungserfolg der Winterlaicher Schnäpel (Coregonus maraena) und Quappe (Lota lota) beeinträchtigen (Thiel 2014). Dagegen könnten höhere Wassertemperaturen in den Sommermonaten das Auftreten von Sauerstoffmangelsituationen begünstigen, was ein zusätzliches Gefährdungspotenzial für die Fischfauna darstellt (Thiel und Thiel 2015). Das sog. Sauerstoffloch wirkt insbesondere für Wanderfischarten als Barriere, die stromauf oder stromab gerichtete Wanderungen verzögern oder sogar unterbrechen könnte (Thiel 2011).

Als Folge des Meeresspiegelanstiegs ist auch eine Verschiebung der oberen Brackwassergrenze stromaufwärts zu erwarten (Schönberg et al. 2014). Eine Zunahme der Anzahl und der Abundanz mariner Fischarten stromauf und eine entsprechende
Abnahme bei limnischen Fischarten stromab wäre eine mögliche Folge (Schönberg et al. 2014). Die Verlagerung des Salinitätsgradienten in der Tideelbe könnte unterhalb des Hamburger Hafens außerdem die Größe der Laichareale und Aufwuchsgebiete bestimmter Arten (z. B. Finte und Schnäpel) verringern.

In welchem Maße sich der Klimawandel zukünftig tatsächlich auf die Fischgemeinschaft der Elbe auswirken wird, wird auch maßgeblich von menschlichen Aktivitäten abhängen, welche die Anpassungsmöglichkeiten der Fische beeinträchtigen (z. B. Ausbau von Fließgewässern, Verengung des Überflutungsraumes durch Eindeichung) oder fördern können (z. B. Errichtung von Fischaufstiegsanlagen, Schaffung neuer oder Anbindung bestehender Flachwasser- und Nebenstromgebiete sowie Anlage neuer Überflutungsräume) (vgl. Schönberg et al. 2014; Thiel und Thiel 2015).

\subsection{Lübecker Bucht}

Im Rahmen des zweiten Klimaberichtes wird erstmals die Lübecker Bucht - das südwestliche Ende der Mecklenburger Bucht in der Ostsee - betrachtet. Im Vergleich zur Nordsee ist die Ostsee gegen Einwirkungen des Atlantischen Ozeans und Gezeiten weitestgehend geschützt. Weite Teile der Ostsee sind permanent geschichtet, und anoxische Bedingungen kommen in den tieferen Teilen regelmäßig vor.

Das Phytoplankton in der Region ist durch zwei Maxima der Chlorophyllkonzentrationen gekennzeichnet. Einmal im März, wenn es zur Kieselalgenfrühjahrsblüte kommt, und zum zweiten im Herbst. Die für die zentrale Ostsee charakteristische Sommerblüte der stickstofffixierenden Blaualgen (Cyanobakterien) tritt nur in sehr geringem Umfang auf. Grund dafür sind die für Ostseeverhältnisse hohen Salzgehalte der Mecklenburger Bucht (mit einem Sommermittel von etwa 11,5 Promille), die sich in deutlich verringerten Wachstumsraten der Stickstofffixierer niederschlagen (Stal et al. 1999). Das Mesozooplankton in der Mecklenburger Bucht ist generell durch einen starken Abundanzrückgang charakterisiert (Wasmund et al. 2011a), wobei insbesondere mikrophage Rotatorien auf dem Rückzug sind. Dies lässt sich in einzelnen Jahren mit kalten und lang anhaltenden Wintern begründen. Hinzu kommt ein Rückgang der Eutrophierung, der sich negativ auf das Mesozooplankton auswirkt (Wasmund et al. 2011a). Auf der anderen Seite zeigt sich ein ansteigender Trend bei Acartia longiremis.

In den flacheren Randgebieten treten submerse Makrophyten auf, wobei Seegras und Blasentang dominieren (Fürhaupter et al. 2008; Mossbauer et al. 2013). Dabei wird ihre Verbreitung in größere Tiefen vor allem durch die geringen Sichttiefen im Sommer verhindert (Duarte 1991; Krause-Jensen et al. 2008), während die Wellenexposition die obere Verbreitungstiefe bestimmt (Koch 2001).

Die benthische Besiedlung der Lübecker Bucht unterteilt sich stark in die Bereiche mit und ohne Sprungschicht. Auf den vorwiegend sandigen Böden im Flachwasser ist eine stellenweise artenreiche Gemeinschaft dokumentiert (Zettler et al. 2000). Hartböden in der aphotischen Zone werden vorwiegend von Miesmuscheln besiedelt. Die Weichbodengemeinschaft un- 
$1981-1986$

Lusitanische Arten

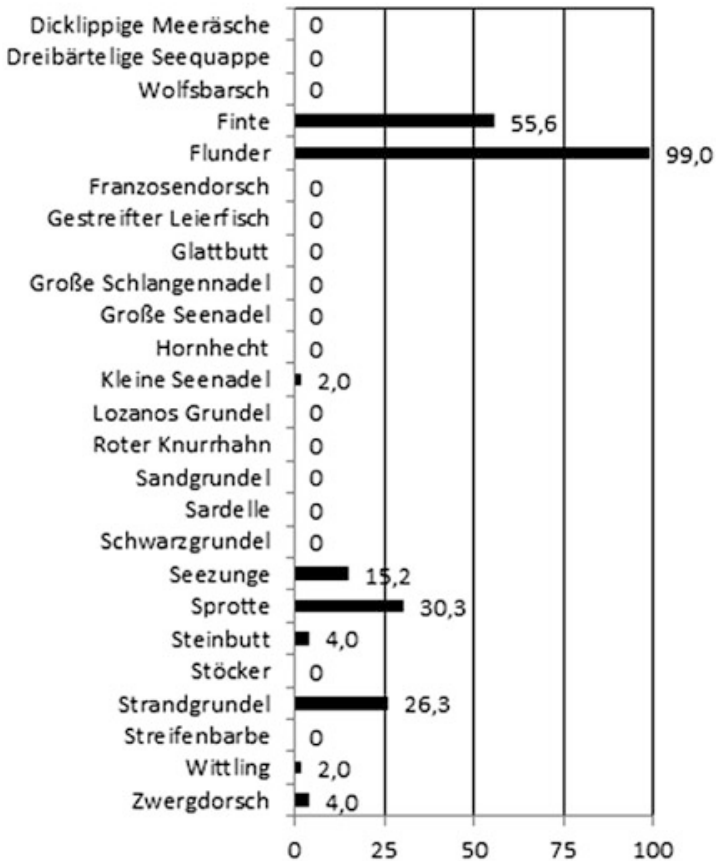

Boreale Arten
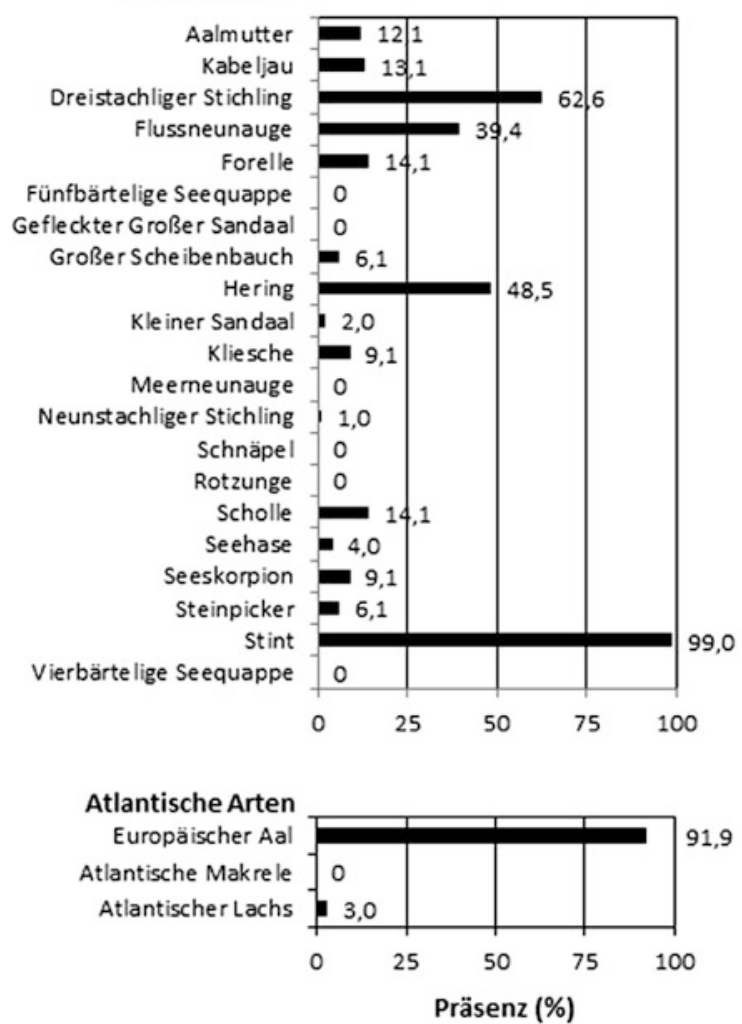

1990 - 1994
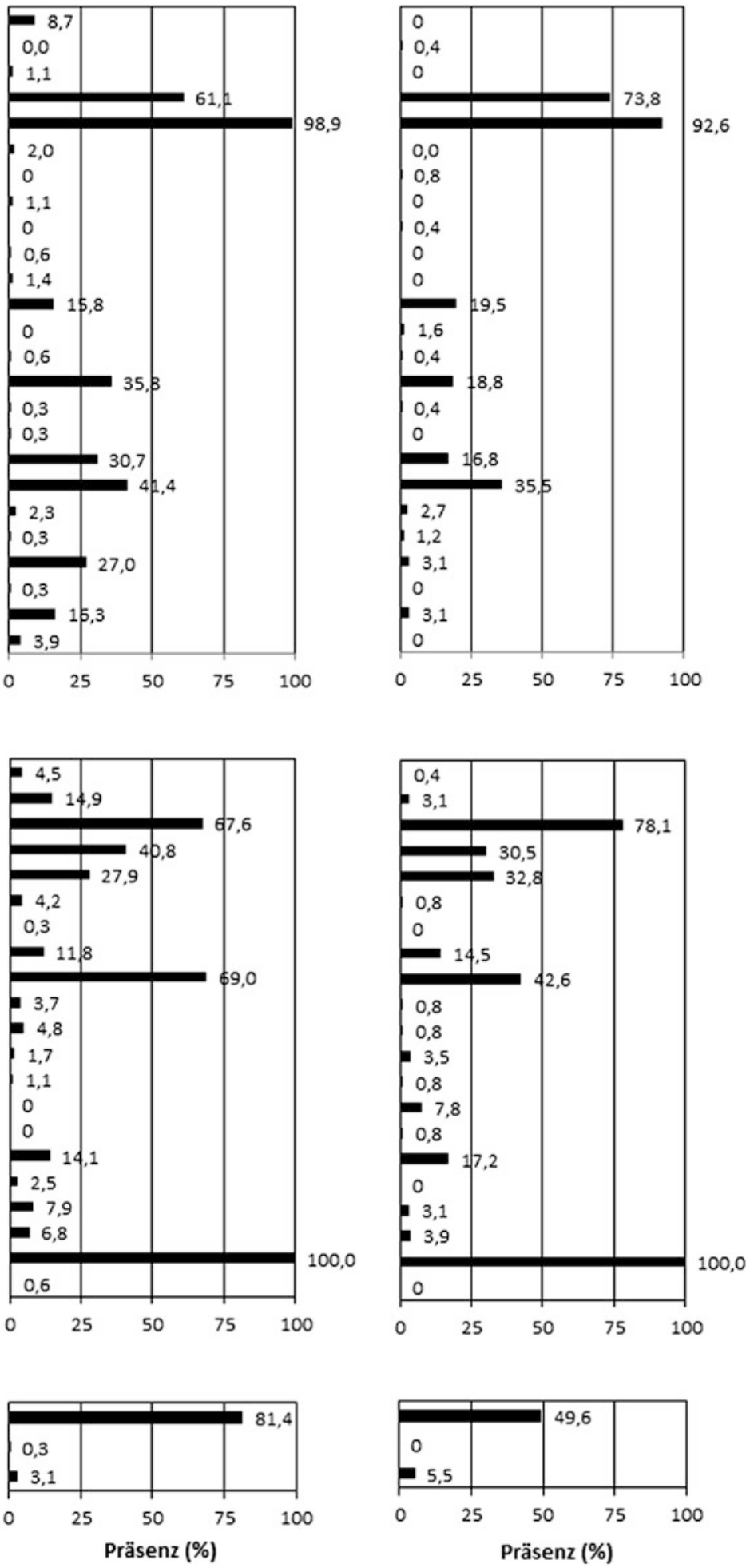

$2009-2010$

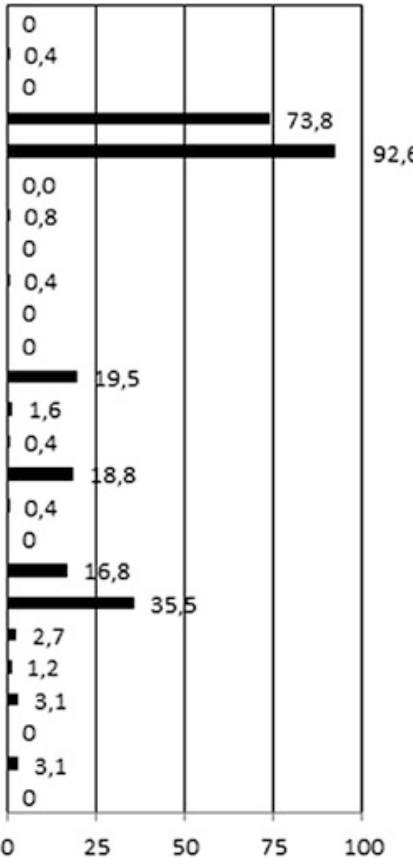

- Abb. 5.7 Präsenzen der mit Hamen im Elbeästuar zwischen Stade und Cuxhaven von 1981 bis 2010 gefangenen Fischarten nach Daten für 1981-1986 von Möller (1984) und Möller (1988), für 1990-1994 von Thiel et al. (1995), Thiel und Potter (2001) und Thiel et al. (2003) sowie für 2009-2010 von Eick und Thiel (2014)

terhalb der Sprungschicht ist dagegen artenarm. Sie wird von einer hohen Fluktuation der Artenzusammensetzung geprägt (Prena et al. 1997) und ist einem permanenten Stress ausgesetzt, der durch die sich im Jahresgang ändernden Sauerstoffkonzentrationen und Salzgehalte induziert wird. Insbesondere in der inneren Lübecker Bucht sind daher die langlebigen Muscheln nahezu verschwunden (Zettler et al. 2000; Schiele et al. 2014). Vor allem opportunistische Borstenwürmer gehören zu den Erstbesiedlern nach den saisonalen anoxischen Phasen (Zettler et al. 2000). 


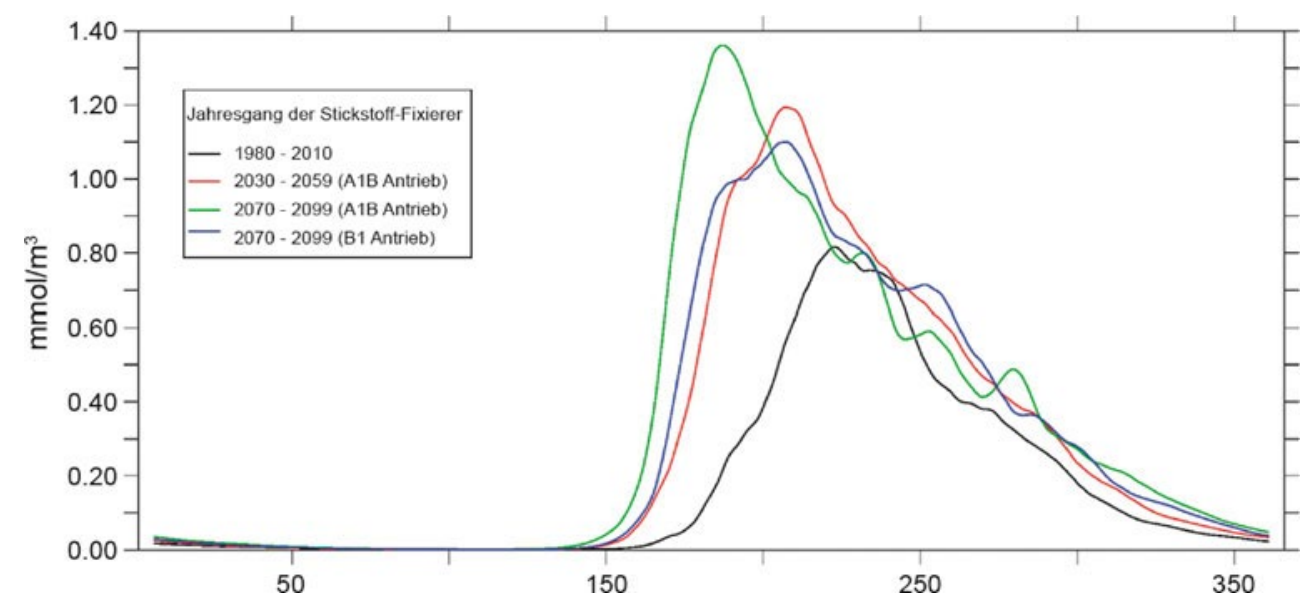

- Abb. 5.8 Stickstofffixierende Blaualgen hängen in ihren Wachstumsbedingungen insbesondere von Temperatur und Salzgehalt ab. Aufgrund der Temperaturzunahme und der Salzgehaltabnahme zeigen die Simulationen, dass sich ihre Blüten bis zum Ende des 21 . Jahrhunderts verstärken und verlängern werden

Im Spätsommer/Frühherbst kommt es regelmäßig zu subtoxischen Bedingungen in Bodennähe (weniger als $2 \mathrm{mg} / \mathrm{l}$ gelöster Sauerstoff), die insbesondere aufgrund der starken Schichtung des Wassers mit Salzgehaltdifferenzen von mehr als 10 Promille zwischen Oberfläche und Bodenwasser entstehen (Wasmund et al. 2011a). Bei Sauerstoffmangel wird Phosphat aus dem Sediment freigesetzt, was wiederum verstärkte Blaualgenblüten nach sich zieht, die nach ihrem Absterben mehr totes organisches Material am Boden und somit eine Verstärkung der sauerstoffverbrauchenden Prozesse hervorrufen (Carstensen et al. 2014). Daneben führen Anoxien zu einer Degradation bis hin zum Absterben der benthischen Fauna, was u. a. einen Rückgang der Bioturbation bewirkt (Josefson et al. 2012).

Es ist davon auszugehen, dass die Mecklenburger Bucht sich aufgrund des Klimawandels in ihren abiotischen und biotischen Gegebenheiten verändern wird, was deutliche Veränderungen des Ökosystems nach sich ziehen wird (BACC Author Team 2015). Die Veränderungen werden aber auch von vielen anderen Faktoren wie der Eutrophierung oder dem Fischereidruck abhängen (Niiranen et al. 2013). Unmittelbar verändern wird sich insbesondere der $\mathrm{pH}$-Wert, der aufgrund des Anstiegs der globalen $\mathrm{CO}_{2}-$ Konzentration der Luft deutlich abnehmen wird (Omstedt et al. 2012). Die Wassertemperaturen werden etwa $2-3 \mathrm{~K}$ bis zum Ende des 21. Jahrhunderts (Friedland et al. 2012) steigen. Die Wahrscheinlichkeit, dass die mittlere Oberflächentemperatur in der Mecklenburger Bucht im Sommer $18^{\circ} \mathrm{C}$ übersteigt, wird sich bis zum Ende des Jahrhunderts verdoppeln (Neumann et al. 2012).

Induziert durch den Temperaturanstieg, kommt es zu einem Rückgang der Eisbildung im Winter. Dies kann ostseeweit dazu führen, dass nur noch die Hälfte bis ein Drittel der heutigen Fläche mit Eis bedeckt wird (Neumann 2010). Die reduzierte Eisbedeckung wird dabei zu Veränderungen der biogeochemischen Kreisläufe führen. So kann sich die winterliche Durchmischung verstärken, ebenso wie die Resuspension des Sedimentes (Eilola et al. 2013).

Die zukünftige Entwicklung des Salzgehaltes ist deutlich unsicherer; eine Abnahme kann im Bereich der Mecklenburger Bucht zwischen 0,5 und 3 Promille betragen (Gräwe et al. 2013; Friedland et al. 2012). Aufgrund des Meeresspiegelanstiegs wird die mit den großen Salzwassereinströmen in die Ostsee transportierte Salzmenge aber größer, die Häufigkeit der Einströme verändert sich dabei nicht (Gräwe et al. 2013) und bleibt ein insbesondere vom Wind abhängiger stochastischer Vorgang.

\subsubsection{Plankton}

Mit einer Abnahme des Salzgehaltes und einer Zunahme der Temperatur (s. auch Abschn. 2.3 und 4.3) werden die Sommerblüten der Stickstofffixierer, die zurzeit in der Mecklenburger Bucht nahe an ihrer Wachstumsgrenze von 12 Promille leben, wahrscheinlich früher anfangen und länger andauern (Friedland et al. 2012; Paerl und Paul 2012; Hense et al. 2013; - Abb. 5.8). In Modellsimulationen kommt es daher zu einer Zunahme der Chlorophyllkonzentrationen in den Sommermonaten, während sich die Frühjahrs- und Herbstblüten nur wenig ändern. Dem widersprechen Mesokosmos-Experimente von Sommer et al. (2012), die eine Beschleunigung der Entwicklung der Frühjahrsblüte beobachtet haben, und auch Eilola et al. (2013), die gezeigt haben, dass der Rückgang der Eisbedeckung zu einem früheren Beginn der Frühjahrsblüte führt. Hier besteht noch Klärungsbedarf. Einen Anstieg der Gesamtprimärproduktion durch das Phytoplankton zeigen alle Ökosystemmodelle (Meier et al. 2012b). In Verbindung mit dem Temperaturanstieg steigt auch die Gefahr, dass potenziell nicht nur toxische Blaualgen deutlich häufiger auftreten, sondern auch pathogene Vibrionen (BakerAustin et al. 2013), die sich bei Wassertemperaturen über $20^{\circ} \mathrm{C}$ verstärkt vermehren.

Aufgrund der Zunahme des Phytoplanktons und der Biomasse im Allgemeinen wird mehr totes organisches Material vorhanden sein. Damit ist auch von einer Verstärkung der sauerstoffzehrenden mikrobiellen Abbauprozesse auszugehen, die wiederum zu einer stärkeren Sauerstoffschuld im Bodenwasser führen wird (Friedland et al. 2012; Meier et al. 2011, 2012a). Da es daneben bei einer Erwärmung und Versüßung des Wasser zu einer Reduktion der Sauerstofflöslichkeit kommen wird (Neumann und Friedland 2011), zeigen die Simulationen (Friedland et al. 2012), dass sich der Zeitraum mit sauerstoffarmen Bedin- 
$200 \%$

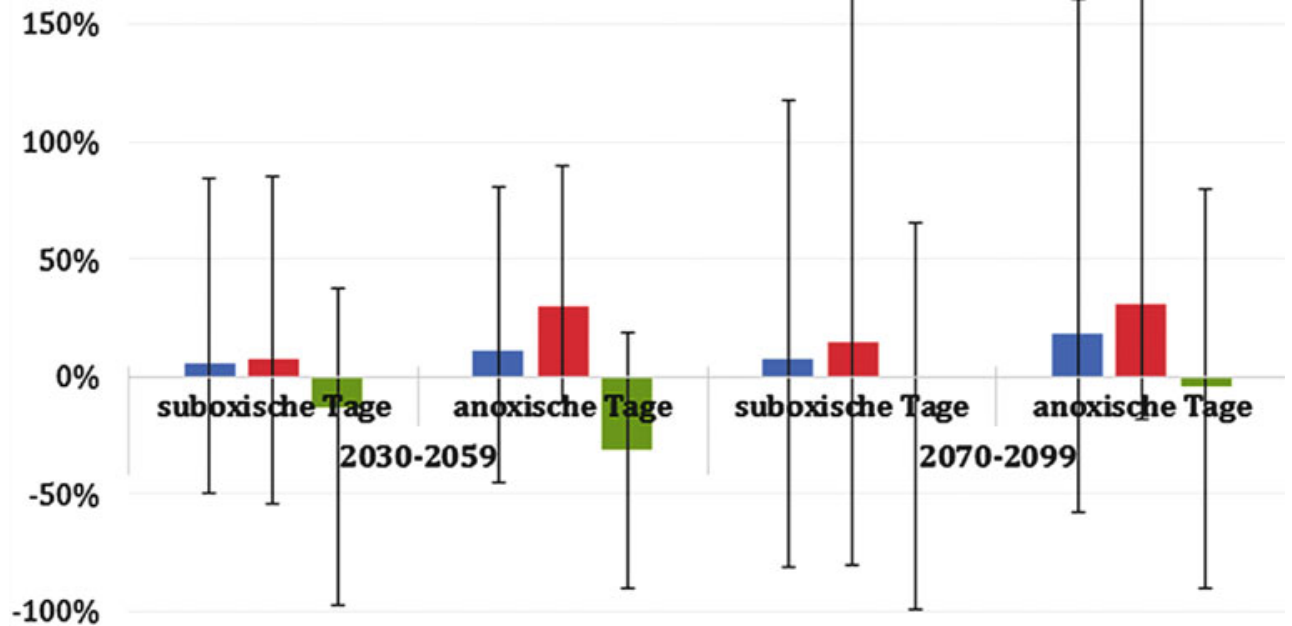

-A1B $=$ A1B_2 $=$ A1B_BSAP

- Abb. 5.9 Die Simulationen des IOW unter Verwendung der zwei Realisierungen des A1B-Szenarios zeigen, wenn sich die Nährstoffeinträge nicht ändern, eine deutliche Zunahme der Tage mit weniger als 2 (0) mg/l Sauerstoff am Boden gegenüber dem Mittel von 1980-2009. Dies geschieht größtenteils bereits bis zur Mitte des 21. Jahrhunderts; danach steigt ihre absolute Anzahl pro Jahr nur noch wenig, aber die Standardabweichung nimmt deutlich zu, d. h., einzelne Ereignisse können deutlich länger als zuvor dauern. Einzig die Umsetzung der Frachtreduktionen entsprechend dem Baltic Sea Action Plan (BSAP, graue Balken) führt zu einem leichten Rückgang des Sauerstoffstresses, wobei sich der Effekt am Ende des 21. Jahrhunderts mit den klimawandelinduzierten Veränderungen ausgleicht, sodass sich die Anzahl der sub- oder anoxischen Tage gegenüber der heutigen Situation kaum verändert

gungen aufgrund des Klimawandels um bis $30 \%$ verlängern kann (- Abb. 5.9). Dabei nimmt die Anzahl der Tage mit anoxischen Bedingungen stärker zu als bei den suboxischen Bedingungen. Zwischen der ersten Hälfte und dem Ende des 21. Jahrhunderts zeigen sich nur noch geringfügige Unterschiede in der mittleren Anzahl der anoxischen/suboxischen Tage, während die Standardabweichung am Ende deutlich zunimmt. Das heißt, dass die Extremsituationen weiter zunehmen werden und sich somit der Sauerstoffstress für die am Boden lebenden Organismen deutlich verstärkten wird.

Dieses Bild ändert sich partiell, wenn man annimmt, dass die Nährstofffrachten in die Ostsee substanziell verringert werden, wie dies im BSAP (HELCOM 2007) vorgesehen ist. Simulationen mit den maximal erlaubten Frachten von 2007 (und auch denen von 2013) in Kombination mit verschiedenen Klimawandelszenarien (Friedland et al. 2012) zeigen jeweils, dass die Frachtreduktionen, die vorrangig auf Phosphor fokussiert sind, Reduktionen der Blaualgenblüten sowohl in der offenen Ostsee als auch in der Mecklenburger Bucht bewirken können. Dies würde dazu führen, dass die anoxischen Bedingungen in der Mecklenburger Bucht auf dem heutigen Niveau verbleiben (- Abb. 5.9) ohne sich weiter zu verschlimmern. Dies wurde u. a. auch von Meier et al. (2012a) berichtet. Einen ähnlichen Effekt sehen Lennartz et al. (2014) in Analysen der Langzeitdaten für Boknis Eck.

\subsubsection{Benthos}

Da in den tieferen Zonen bereits heute schon Organismengruppen dominieren, die an Sauerstoffstress angepasst sind (Schiele et al. 2014), kann es sein, dass der Klimawandel insgesamt nur wenig Veränderungen der Benthos-Artengemeinschaften nach sich ziehen wird. Die Verringerung des Salzgehaltes in Kombination mit den verschlechterten Sauerstoffbedingungen kann aber zu einer Verringerung der Biodiversität oder zumindest zu einem Rückgang ihrer Biomasse führen, da zurzeit bereits viele Arten an ihrem Salzgehalttoleranzlimit vorkommen (z. B. Arctica islandica, RADOST 2014). Eine weitere Reduktion des Salzgehaltes kann in Kombination mit den zusätzlichen prognostizierten Veränderungen des Klimawandels (Temperatur, pH-Wert) zu erhöhtem Dauerstress für die Islandmuschel führen, sodass ihre Stressresistenz weiter sinkt. In den Randbereichen der Lübecker Bucht kann es zukünftig zu anoxischen Ereignissen kommen, die bislang nur selten oder nie aufgetreten sind, und damit zu einer Verschiebung der Artengemeinschaft von langlebigen Arten zu kurzlebigen (z. B. Meeresborstenwürmer, RADOST 2014).

Ein etwas überraschender Zusammenhang wurde zwischen der Eisbedeckung und der Entwicklung der Muschelbiomasse hergestellt (RADOST 2014). So stellt das Eis zurzeit einen Schutz der Muscheln gegenüber dem Fraß durch die Eisente dar. Nimmt die Eisbedeckung ab, verstärkt sich also der Fraßdruck durch die Eisente, sodass mit einem Rückgang der winterlichen Muschelbiomasse zu rechnen ist.

Die Blasentang- und Seegrasvorkommen in der Lübecker Bucht sind zwar auf der einen Seite relativ tolerant gegenüber Veränderungen der äußeren Bedingungen, insbesondere gegenüber den prognostizierten Salzgehalt- und Temperaturveränderungen (Bobsien 2014). Aber ob sie sich evolutionär an die durch den Klimawandel induzierten Veränderungen ausreichend schnell anpassen können, ist offen. Kritisch sind für sie vor allem die Entwicklung der Sichttiefe und die Sauerstoffsituation, da insbesondere Seegras anoxische Bedingungen in der Wassersäule über Nacht nur sehr kurz toleriert (Bobsien 2014). Zudem belasten extreme Ereignisse wie Hitzewellen die Makrophyten. 
Insbesondere die Kombination von hohen Wassertemperaturen, geringer Lichtverfügbarkeit und sauerstofffreiem Wasser mit hohen Schwefelwasserstoffkonzentrationen in der Wassersäule und im Sediment können Seegraswiesen nachhaltig schädigen (Moore und Jarvis 2008; Höffle et al. 2011). So stellen für den Blasentang bereits Wassertemperaturen über $20^{\circ} \mathrm{C}$ einen Stressfaktor dar - ohne jedoch letal zu sein. Gleichzeitig steigt dann die Sensitivität gegenüber weiteren Stressoren (Wahl et al. 2011).

Aufgrund der Veränderungen im Jahresgang des Phytoplanktons kommt es zu einem deutlichen Rückgang der mittleren Sichttiefe bzw. der Klarwasserphase im Juni und Juli. Zur Verbesserung der Wachstumsbedingungen wurde daher im Rahmen des BSAP festgelegt, dass in der Mecklenburger Bucht die Sichttiefe mindestens 7,43 m betragen soll (Tab. F1 bei HELCOM 2013). Friedland et al. (2012) zeigen, dass die Umsetzung des BSAP Verbesserungen der Sichttiefe von bis $\mathrm{zu} 2 \mathrm{~m}$ nach sich ziehen können, was entsprechend Mossbauer et al. (2013) eine Verdoppelung der potenziell geeigneten Siedlungsgebiete für submerse Makrophyten bewirken kann.

Die fehlende Eisbedeckung kann dazu führen, dass die mechanische Beanspruchung emerser Makrophyten deutlich abnimmt (Friedland et al. 2012), wobei offen ist, ob sie nur positiv auf die fehlende Eisbedeckung reagieren, da dies ihrer bisherigen evolutionären Entwicklung entgegenläuft. So kann die Eisbedeckung auch einen Schutz vor Fraßfeinden darstellen.

\subsubsection{Fische}

Auch in der Ostsee muss der Klimawandel in seiner Wirkung auf Fische stets in Kombination mit anderen Faktoren betrachtet werden. Die Interaktion zwischen Klimaveränderungen (Anstieg von Temperatur- und Sauerstoffstress, Rückgang des Salzgehaltes und ph-Wertes) und Fischerei kann weitreichende Auswirkungen auf die Fischpopulationen haben (Peltonen et al. 2012).

Das generelle Muster einer nordwärtigen Verschiebung der geografischen Artverbreitung im Atlantik wird vom Salinitätsgradienten überlagert, der die Verbreitung mariner Arten begrenzt. So existiert sowohl im Kattegatt als auch in der südlichen Ostsee eine erhöhte Artenvielfalt (Hiddink und Coleby 2012), u. a. durch Sardellen, die seit den 1990er-Jahren in der südlichen Ostsee reproduzieren (Alheit et al. 2012). Der verfügbare Lebensraum mariner Fischarten könnte sich ostseeweit verkleinern, während süßwasseradaptierte Arten potenzielles Verbreitungsgebiet hinzugewännen (MacKenzie et al. 2007).

Möllmann et al. (2009) beschreiben zwei alternative Zustände der Fisch- und Zooplanktonzönosen in der zentralen Ostsee, bei denen entweder Dorsch und Pseudocalanus acuspes oder Sprotte und Acartia spp. dominieren. Welche der beiden Ausprägungen dominant ist, hängt dabei von natürlichen und anthropogenen Faktoren ab, wozu auch die atmosphärischen Klimabedingungen zählen. So kam es zwischen 1988 und 1993 zu einem Umschlagen vom Dorsch- zum Sprottenregime bzw. von Pseudocalanus zu Acartia (einem sog. Regimeshift), wobei die abiotischen Eigenschaften der Ostsee in dieser Zeit mit geringen Salz- und Sauerstoffgehalten und relativ hohen Wassertemperaturen den prognostizierten Bedingungen entsprachen (Meier 2015). Zusätzlich wird für Acartia spp. berichtet (Otto et al. 2014), dass sie auf erhöhte Frühjahrstemperaturen positiv reagieren und es somit zu einem Anstieg ihrer Biomasse kommen kann, d. h., das Sprotten- bzw. Acartia-Regime wäre zukünftig weiter dominant.

Durch eine frühzeitige Erwärmung des Wassers kann aber auch die Synchronizität zwischen der temperaturgesteuerten Zooplanktonentwicklung und der tageslängengesteuerten Phytoplanktonentwicklung verringert werden. Dies hätte negative Konsequenzen für die Nahrungsversorgung planktivorer Fische. Beispielsweise sind Heringslarven nach Aufbrauchen ihres Dottervorrats auf eine ausreichende Zooplanktondichte angewiesen, was durch einen zunehmenden Mismatch zwischen der Phyto- und Zooplanktonentwicklung jedoch beeinträchtigt wäre (Houde 2009; Polte et al. 2013).

Die prognostizierten Temperaturveränderungen wirken sich auf die Planktonentwicklung (Dahlgren et al. 2011; Wasmund et al. 2011b) und die Reproduktion der Fische (Margonski et al. 2010) aus und können auf diesem Wege schließlich die Nahrungsverfügbarkeit der Endglieder der trophischen Kaskade wie Seevögel beeinflussen. Der Klimawandel ist jedoch nur einer unter vielen Faktoren, welche die Fischpopulationen der Ostsee beeinflussen. So kann die Entwicklung der Fischbestände in der Ostsee zwischen 1900 und 1980 größtenteils durch den Rückgang der Robbenpopulation (und damit die fehlende Top-down-Kontrolle) und die starke Eutrophierung erklärt werden (Österblom et al. 2007).

\section{$5.6 \quad$ Fazit}

Die Dynamik der Ökosysteme der Nordsee, des Wattenmeeres, des Elbeästuars und der Lübecker Bucht werden alle durch klimabedingte Faktoren wie Temperatur, Niederschlag oder Meeresströmungen beeinflusst. Die tatsächliche Auswirkung ist aber meistens komplex, weil mehrere natürliche und anthropogene Faktoren interagieren. Die anthropogenen Faktoren sind an der Küste am größten und nehmen zum offenen Meer ab, während der Einfluss klimaabhängiger Faktoren wie Temperatur oder Meeresströmungen zunimmt.

Steigende Wassertemperaturen, zunehmende atlantische Einflüsse und Fischerei haben eine deutliche Auswirkung auf das Ökosystem der Nordsee. Das Plankton entwickelt sich durch bessere Lichtbedingungen und höhere Temperaturen früher im Jahr. Makrobenthos und Fischfauna reagieren auf höhere Temperaturen durch eine Zunahme südlicher wärmeliebender und eine Abnahme kälteliebender Arten. Höhere Temperaturen haben die Reproduktionszeiten des Makrobenthos verlängert, nicht aber die Ernährungstypen des Makrobenthos durch verändertes Nahrungsangebot geändert. Wie die Wechselwirkung von Klimawandel und Fischerei sich auf die Fischfauna auswirken wird, ist unklar.

Temperatur und Nährstofffrachten prägten die Langzeitdynamik des Wattenmeeres während der letzten Dekaden. In einem wärmeren Wattenmeer können schnellere Stoffumsätze insbesondere des Phosphats zu einer erhöhten Produktivität führen. Weiter sinkende Nährstofffrachten der Flüsse infolge der Umsetzung von EU-Umweltgesetzen werden dem entgegenwirken und unklar ist, wie die Klimaänderung die Abflussmengen und damit auch die Nährstofffrachten ändern wird. Langfristig werden sich heimische kälteliebende Arten in höhere Breiten zurückziehen, weitere Or- 
ganismen aus südlicheren Gebieten ihr Verbreitungsareal nach Norden ausdehnen, und eingeschleppte Arten von wärmeren Küsten werden sich weiterhin etablieren. Offen ist nach wie vor die Frage, ob das Wattenmeer genügend Sediment aus der Nordsee importieren kann, um mit dem Anstieg des Meeresspiegels Schritt halten zu können. Sollte das nicht der Fall sein, werden wichtige Habitate wie Gezeitenflächen verschwinden, und das Ökosystem des Wattenmeeres wird sich grundlegend ändern.

Im stark von menschlichen Eingriffen geprägten Elbeästuar sind klimabedingte Änderungen schwer zu erkennen. Klar ist aber, dass ein Temperaturanstieg die jetzige Sauerstoffproblematik im Hamburger Hafenbereich verstärken wird. Wie im letzten Klimabericht schon festgestellt, fehlen rezente Forschungsergebnisse, um eine fundierte Aussage über die Änderung des Planktonsystems im Elbeästuar bzgl. Klimaänderung treffen zu können. Es gibt zwar eine Reihe von Beobachtungen aus anderen Ästuaren, die Hinweise auf mögliche Änderungen geben. Es sei aber festzuhalten, dass ästuarine Systeme schwer zu vergleichen sind, weil die Phänologie der Planktondynamik stark von lokalen Effekten geprägt wird. Die bisher vorhandene Datenbasis reicht ebenfalls noch nicht aus, belastbare Aussagen über den Einfluss des Klimawandels auf das Makrozoobenthos in der Tideelbe zu treffen. Es ist aber anzunehmen, dass die Zahl eingeschleppter Arten zunehmen wird. Eine Zunahme der Sauerstoffmangelsituationen wird die Fischwanderungen beeinträchtigen, Änderungen des Salinitätsgradienten können zur Verringerung von Laicharealen und Aufwuchsgebieten bestimmter Arten führen. In welchem Maße sich der Klimawandel zukünftig tatsächlich auf die Fischgemeinschaft der Elbe auswirken wird, wird auch maßgeblich von menschlichen Aktivitäten abhängen, welche die Anpassungsmöglichkeiten der Fische beeinträchtigen oder fördern können.

Neu im 2. HKB ist die Einbeziehung der Ostsee/Lübecker Bucht. Eine Erwärmung wird zu weniger Eisbedeckung im Winter und wärmeren Temperaturen im Sommer führen. Die Folgen wären frühere Algenblüten im Frühjahr sowie eine höhere Phytoplanktonproduktivität und mehr Blaualgen im Sommer. Höhere Produktivität und Temperaturen führen im Prinzip zu weniger Sauerstoff im Bodenwasser. Das Makrobenthos der Ostsee ist an solche Bedingungen angepasst. Sollte es aber zu Sauerstofflosigkeit kommen, wird sich die Zusammensetzung des Makrobenthos hin zu kurz lebenden Formen verschieben. Wie im Wattenmeer und Nordsee ist die zukünftige anthropogene Belastung mit Nährstoffen unklar.

Die in diesem Klimabericht dargestellten neuen Erkenntnisse über klimabedingte Änderungen in aquatischen Ökosystemen bestätigen die Ergebnisse des 1. HKB, dass insbesondere die Wassertemperatur und Hydrodynamik die Variabilität der Ökosysteme prägen, während hin zur Küste Interaktionen mit anthropogenen Faktoren zugenommen haben und vermutlich die zukünftige ökologische Entwicklung prägen werden. Ein Vergleich der im 1. HKB erwähnten möglichen Auswirkungen des Klimawandels auf die ökologische Entwicklung mit neuen Erkenntnissen unterstreicht aber die Schwierigkeit von Vorhersagen. So wurden eine zunehmende Dominanz von Quallen und negative Effekte der Versauerung auf die Planktongemeinschaft erwartet. Beide Vorhersagen sind (noch) nicht eingetroffen. Die Beispiele machen den Bedarf an weiteren Langzeitbeobachtungen und Begleitforschung klar.

\section{Literatur}

Ahlhorn F (2009) Long-term perspective in coastal zone development. Springer, Heidelberg

Alheit J, Bakun A (2010) Population synchronies within and between ocean basins: Apparent teleconnections and implications as to physical-biological linkage mechanisms. J Marine Syst 79:267-285

Alheit J, Pohlmann T, Casini M, Greve W, Hinrichs R, Mathis M, O'Driscoll K, Vorberg R, Wagner C (2012) Climate variability drives anchovies and sardines into the North and Baltic Seas. Prog Oceanogr 96:128-139

Alvarez-Fernandez S, Lindeboom H, Meesters E (2012) Temporal changes in plankton of the North Sea: community shifts and environmental drivers. Mar Ecol-prog Ser 462:21-38

Amann T, Weiss A, Hartmann J (2012) Carbon dynamics in the freshwater part of the Elbe estuary, Germany: implications of improving water quality. Estuar Coast Shelf S 107:112-121

Andersen HE, Kronvang B, Larsen SE, Hoffmann CC, Jensen TS, Rasmussen EK (2006) Climate-change impacts on hydrology and nutrients in a Danish lowland river basin. Sci Total Environ 365:223-237

Aprahamian MW, Aprahamian CD, Knights AM (2010) Climate change and the green energy paradox: the consequences for twaite shad Alosa fallax from the River Severn, UK. J Fish Biol 77:1912-1930

Aurich HJ (1953) Verbreitung und Laichverhältnisse von Sardelle und Sardine in der südöstlichen Nordsee und ihre Veränderungen als Folge der Klimaänderung. Helgoländer Wissensch Meeresunters 4:175-204

Author Team BACC II (2015) Second Assessment of climate change for the Baltic Sea basin. Regional climate studies. Springer, Berlin

Baker-Austin C, Trinanes JA, Taylor NGH, Hartnell R, Siitonen A, Martinez-Urtaza J (2013) Emerging Vibrio risk at high latitudes in response to ocean warming. Nat Clim Change 3:73-77

Barbier EB, Hacker SD, Kennedy C, Koch EW, Stier AC, Silliman BR (2011) The value of estuarine and coastal ecosystem services. Ecol Monogr 81:169-193

Beaugrand G (2009) Decadal changes in climate and ecosystems in the North Atlantic Ocean and adjacent seas. Deep Sea Research II 56:656-673

Beaugrand G, McQuatters-Gollop A, Edwards M, Goberville E (2013) Long-term responses of North Atlantic calcifying plankton to climate change. Nat Clim Change 3:263-267

Beck M, Brumsack HJ (2012) Biogeochemical cycles in sediment and water column of the Wadden sea: the example Spiekeroog island in a regional context. Ocean Coast Manage 68:102-113

Becker J, Ortmann C, Wetzel MA, Koop JH (2015) Metabolic activity and behavior of the invasive amphipod Dikerogammarus villosus and two common Central European gammarid species (Gammarus fossarum, G. roeselii): Low metabolic rates may favor the invader. Comp Biochem Physiol A Mol Integr Physiol 191:119-126

Berkenhagen J, Döring R, Fock HO, Kloppmann MHF, Pedersen SA, Schulze T (2010) Decision bias in marine spatial planning of offshore wind farms: Problems of singular versus cumulative assessments of economic impacts on fisheries. Mar Pol 34:733-736

van Beusekom JEE, Brockmann UH (1998) Transformation of phosphorus in the Elbe estuary. Estuaries 21:518-526

van Beusekom JEE, Loebl M, Martens P (2009a) Distant riverine nutrient supply and local temperature drive the long-term phytoplankton development in a temperate coastal basin. J Sea Res 61:26-33

van Beusekom JEE, Bot P, Carstensen J, Goebel J, Lenhart H, Pätsch J, Petenati T, Raabe T, Reise K, Wetsteijn B (2009b) Eutrophication. Thematic Report No. 6. In: Marencic H, de Vlas J (Hrsg) Quality Status Report 2009, WaddenSea Ecosystem. No 25. Common Wadden Sea Secretariat, Trilateral Monitoring and Assessment Group, Wilhelmshaven

Bianchi CN (1997) Climate change and biological response in the marine benthos. In: Proceedings of the Italian Association for Oceanology and Limnology, S 3-20

Bobsien I (2014) Mögliche Auswirkungen des Klimawandels auf den Blasentang (Fucus vesiculosus) und das Gewöhnliche Seegras (Zostera marina) in der Ostsee. RADOST-Berichtsreihe Nr. 24.

Boddeke R, Vingerhoed B (1996) The anchovy returns to the Wadden Sea. Ic J seMar Sci 53:1003-1007

Boersma M, Wiltshire KH, Kong SM, Greve W, Renz J (2015) Long-term change in the copepod community in the southern German Bight. J Sea Res 101:41-50 
Brierley AS, Kingsford MJ (2009) Impacts of climate change on marine organisms and ecosystems. Curr Biol 19:R602-R614

Bronstert A (1995) River flooding in Germany: Influenced by climate change? Phys Chem Earth 20:445-450

Buschbaum C, Reise K (2010) Globalisierung unter Wasser - Neues Leben im Weltnaturerbe Wattenmeer. Biol Zeit 3:202-210

Buschbaum C, Lackschewitz D, Reise K (2012) Nonnative macrobenthos in the Wadden Sea ecosystem. Ocean Coast Manage 68:89-101

Cabaco S, Santos R (2007) Effects of burial and erosion on the seagrass Zostera noltii. J Exp Mar Biol Ecol 340:204-212

Cadée GC, Hegeman J (2002) Phytoplankton in the Marsdiep at the end of the $20^{\text {th }}$ century; 30 years monitoring biomass, primary production, and Phaeocystis blooms. J Sea Res 48:97-110

Carstensen J, Conley DJ, Bonsdorff E, Gustafsson BG, Hietanen S, Janas U, Jilbert T, Maximov A, Norkko A, Norkko J, Reed DC, Slomp CP, Timmermann K, Voss M (2014) Hypoxia in the baltic sea: biogeochemical cycles, benthic fauna, and management. AMBIO 43(1):26-36

Cloern JE, Jassby SD (2010) Patterns and scales of phytoplankton variability in estuarine-coastal ecosystems. Estuar Coast 33:230-241

Colijn F, Fanger HU (2011) Klimabedingte Änderungen in aquatischen Ökosystemen: Elbe, Wattenmeer und Nordsee. In: von Storch $\mathrm{H}$, Claussen M (Hrsg) Klimabericht für die Metropolregion Hamburg. Springer, Berlin

Dahlgren K, Eriksson Wiklund AK, Andersson A (2011) The influence of autotrophy, heterotrophy and temperature on pelagic food web efficiency in a brackish water system. Aquat Ecol 45:307-323

Dippner JW, Kröncke I (2015) Ecological forecasting in the presence of abrupt regime shifts. J Marine Syst 150:34-40

Dippner JW, Müller C, Kröncke I (2014) Loss of persistence of the North Atlantic Oscillation and its biological implication. Front Ecol Evol 2(57):1-8

Doody JP (2008) Saltmarsh conservation, management and restoration. Springer, Dordrecht

Drabble R (2012) Monitoring of East channel dredge areas benthic fish population and its implications. Mar Pollut Bull 64:363-372

Duarte CM (1991) Seagrass depth limits. Aquat Bot 363:363-377

Dulvy NK, Rogers SI, Jennings S, Stelzenmüller V, Dye SR, Skjoldal HR (2008) Climate change and deepening of the North Sea fish assemblage: a biotic indicator of regional warming. J Appl Ecol 45:1029-1039

EEA (2015) State of the Europe's seas. EEA Report No 2/2015. European Environment Agency. Publications Office of the European Union, Luxembourg (Webseite der European Environment Agency)

Ehrich S, Adlerstein S, Brockmann U, Floeter JU, Garthe S, Hinz H, Kröncke I, Neumann H, Reiss H, Sell AF, Stein M, Stelzenmüller V, Stransky C, Temming A, Wegner G, Zauke GP (2007) 20 years of the German Small-scale Bottom Trawl Survey (GSBTS): a review. Senckenb Maritima 37:13-82

Eick D, Thiel R (2014) Fish assemblage patterns in the Elbe estuary: guild composition, spatial and temporal structure, and influence of environmental factors. Mar Biodivers 44(4):559-580

Eilola K, Mårtensson S, Meier HEM (2013) Modelling the impact of reduced sea ice cover in future climate on the Baltic Sea biogeochemistry. Geophys Res Lett 40(1):149-154

Emeis KC, van Beusekom J, Callies U, Ebinghaus R, Kannen A, Kraus G, Kröncke I, Lenhart H, Lorkowski I, Matthias V, Möllmann C, Pätsch J, Scharfe M, Thomas H, Weisse R, Zorita E (2015) The North Sea - a shelf sea in the anthropocene. J Mar Syst 141:18-33

Engelhard GH, Elis JR, Payne MR, Ter Hofstede Pinnegar RJK (2011) Ecotypes as a concept for exploring responses to climate change in fish assemblages. ICES J Mar Sci 68(3):580-591

Fast T, Kies L (1990) Biomass and production of phytoplankton in the Elbe estuary. In: Michaelis W (Hrsg) Estuarine water quality management. Springer, Berlin, S 395-398

Fauchald P (2010) Predator-prey reversal: a possible mechanism for ecosystem hysteresis in the North Sea. Ecology 91:2191-2197

Freund JA, Grüner N, Brüse S, Wiltshire KH (2012) Changes in the phytoplankton community at Helgoland, North Sea: Lessons from single spot time series analyses. Mar Biol 159:2561-2571

Friedland R, Neumann T, Schernewski G (2012) Climate change and the Baltic Sea action plan: model simulations on the future of the Western Baltic Sea. J Mar Syst 105-108:175-186
Fulweiler RW, Nixon SW (2009) Responses of benthic-pelagic coupling to climate change in a temperate estuary. In: Andersen JH, Conley DJ (Hrsg) Eutrophication in coastal ecosystems, developments in hydrobiology. Springer, Drodrecht, S 147-156

Fürhaupter K, Wilken H, Grage A, Meyer T (2008) Kartierung der marinen Pflanzenbestände im Flachwasser der Ostseeküste - Schwerpunkt Fucus und Zostera. Bericht des Landesamtes für Natur und Umwelt des Landes Schleswig-Holstein (LANU)

Gambill M, Peck MA (2014) Respiration rates of the polyps of four jellyfish species: Potential thermal triggers and limits. J Exp Mar Biol Ecol 459:17-22

Gasparini S, Castel J, Irigoien X (1999) Impact of suspended particulate matter on egg production of the estuarine copepod, Eurytemora affinis. J Mar Syst 22:195-205

Gebühr C, Wiltshire KH, Aberle N, van Beusekom JEE, Gerdts G (2009) Influence of nutrients, temperature, light and salinity on the occurrence of Paralia sulcata at Helgoland Roads. North Sea Aquat Biol 7:185-197

Gentsch E, Kreibich T, Hagen W, Niehoff B (2009) Dietary shifts in the copepod Temora longicornis during spring: evidence from stable isotope signatures, fatty acid biomarkers and feeding experiments. J Plankton Res 31:45-60

van der Graaf S, Jonker I, Herlyn M, Kohlus J, Fogh Vinther H, Reise K, de Jong D, Dolch T, Bruntse G, de Vlas J (2009) Seagrass. Thematic report. In: Marencic H, de Vlas J (Hrsg) Quality status report 2009. Waddensea ecosystem no. 25, Bd. 12. Common Wadden Sea Secretariat.Trilateral Monitoring and Assessment Group, Wilhelmshaven

Gräwe U, Friedland R, Burchard H (2013) The future of the Western Baltic Sea: two possible scenarios. Ocean Dynam 63(8):901-921

Gröger JP, Kruse GH, Rohlf N (2010) Slave to the rhythm: how large-scale climate cycles trigger herring (Clupea harengus) regeneration in the North Sea. ICES J Mar Sci 67(3):454-465

Heessen H, Daan N, Ellis J (2015) Trawl surveys. In: Heessen HJL, Daan N, Ellis JR (Hrsg) Fish atlas of the Celtic Sea, North Sea and Baltic Sea. KNNV Publishing, Wageningen, S 31-40

Heincke F (1894) Die Fische Helgolands. Beitr Meeresfauna Helgol 1:93-120

HELCOM (2007) HELCOM Baltic Sea action plan. HELCOM Ministerial Meeting, Krakow. Helsinki Commission (HELCOM), Helsinki, S 1-101

HELCOM (2013) Approaches and methods for eutrophication target setting in the Baltic Sea region. Balt Sea Environ Proc 133:1-134

Hempel M, Thiel R (2013) First records of the round goby Neogobius melanostomus (Pallas, 1814) in the Elbe River, Germany. Bioinvasions Rec 2(4):291-295

Henderson PA, Corps M (1997) The role of temperature and cannibalism in interannual recruitment variation of bass in British waters. J Fish Biol 50:280-295

Hense I, Meier HEM, Sonntag S (2013) Projected climate change impact on Baltic Sea cyanobacteria. Clim Chang 119(2):391-406

Hiddink JG, Coleby C (2012) What is the effect of climate change on marine fish biodiversity in an area of low connectivity, the Baltic Sea? Global Ecol Biogeogr 21(6):637-646

Hislop J, Bergstad OA, Jakobsen T, Sparholt H, Blasdale T, Wright P, Kloppmann MHF, Hillgruber N, Heessen H (2015) 32. Cod fishes (Gadidae). In: Heessen H, Daan N, Ellis JR (Hrsg) Fish atlas of the Celtic Sea, North Sea, and Baltic Sea: based on international research-vessel surveys. Academic Publ, Wageningen, S 186-194

Höffle H, Thomsen MS, Holmer M (2011) High mortality of Zostera marina under high temperature regimes but minor effects of the invasive macroalgae Gracilaria verminculophylla. Estuar Coast Shelf Sci 92:35-46

Hollowed AB, Barange M, Beamish RJ, Brander K, Cochrane K, Drinkwater K, Foreman MGG, Hare JA, Holt J, Ito S, Kim S, King JR, Loeng H, MacKenzie BR, Mueter FJ, Okey TA, Peck MA, Radchenko VI, Rice JC, Schirripa MJ, Yatsu A, Yamanaka $Y$ (2013) Projected impacts of climate change on marine fish and fisheries. ICES J Mar Sci 70:1023-1037

Houde ED (2009) Recruitment variability. In: Jakobsen T, Fogarty MJ, Megrey BA, Moksness E (Hrsg) Fish reproductive biology: implications for assessment and management. Wiley-Blackwell, West Sussex, S 91-171

Huang SC, Krysanova V, Osterle H, Hattermann FF (2010) Simulation of spatiotemporal dynamics of water fluxes in Germany under climate change. Hydrol Process 24:3289-3306

IPCC (2014) Climate change 2014: Impacts, Adaptation and Vulnerability. Working Group II Contribution to the Fifth Assessment Report of the Intergo- 
vernmental Panel on Climate Change. Field et al. (Hrsg). Cambridge University Press, Cambridge

de Jonge VN, de Boer WF, de Jong DJ, Brauer VS (2012) Long-term mean annual microphytobenthos chlorophyll a variation correlates with air temperature. Mar Ecol Prog Ser 468:43

de Jonge VN, Schuttelaars HM, van Beusekom JEE, Talke SA, de Swart HE (2014) The influence of channel deepening on estuarine turbidity levels and dynamics, as exemplified by the Ems estuary. Estuar Coast Shelf Sci 139:46-59

Josefson AB, Norkko J, Norkko A (2012) Burial and decomposition of plant pigments in surface sediments of the Baltic Sea - role of oxygen and benthic fauna. Mar Ecol Prog Ser 455:33-49

Keller AA, Oviatt CA, Walker HA, Hawk JD (1999) Predicted impacts of elevated temperature on the magnitude of the winter-spring phytoplankton bloom in temperate coastal waters: a mesocosm study. Limnol Oceanogr 44:344-356

Kennish MJ (2002) Environmental threats and environmental future of estuaries. Environ Conserv 29:78-107

Kirby RR, Beaugrand G, Lindley JA (2009) Synergistic effects of climate and fishing in a marine ecosystem. Ecosystems 12(4):548-561

Kirwan ML, Megonigal JP (2013) Tidal wetland stability in the face of human impacts and sea-level rise. Nature 504:53-60

Koch EM (2001) Beyond light: physical, geological, and geochemical parameters as possible submersed aquatic vegetation habitat requirements. Estuaries 24(1):1-17

Kraberg AC, Carstens K, Peters S, Tilly K, Wiltshire KH (2012) The diatom Mediopyxis helysia at Helgoland Roads: a success story? Helgol Mar Res 66:463-468

Krause-Jensen D, Sagert S, Schubert H, Boström C (2008) Empirical relationships linking distribution and abundance of marine vegetation to eutrophication. Ecol Indic 8:515-529

Kröncke I (2011) Changes in Dogger Bank macrofauna communities in the $20^{\text {th }}$ century caused by fishing and climate. Estuar Coast Shelf Sci 94(3):234-245

Lackschewitz D, Reise K, Buschbaum C, Karez R (2015) Neobiota in deutschen Küstengewässern. Schriftenreihe Landesamt für Landwirtschaft, Umwelt und ländliche Räume Schleswig-Holstein (LLUR)

Lennartz ST, Lehmann A, Herrford J, Malien F, Hansen HP, Biester H, Bange HW (2014) Long-term trends at the Boknis Eck time series station (Baltic Sea), 1957-2013: Does climate change counteract the decline in eutrophication? Biogeosciences 11:6323-6339

Lohmann G, Wiltshire KH (2012) Winter atmospheric circulation signature for the timing of the spring bloom of diatoms in the North Sea. Mar Biol 159:2573-2581

Ly J, Philippart CJ, Kromkamp JC (2014) Phosphorus limitation during a phytoplankton spring bloom in the western Dutch Wadden Sea. J Sea Res 88:109-120

Mackas DL, Greve W, Edwards M, Chiba S, Tadokoro K, Eloire D, Mazzocchi MG, Batten S, Richardson AJ, Johnson C, Head E, Conversi A, Peluso T (2012) Changing zooplankton seasonality in a changing ocean: comparing time series of zooplankton phenology. Prog Oceanogr 97-100:31-62

MacKenzie BR, Gislason H, Möllmann C, Kösters FW (2007) Impact of 21st century climate change on the Baltic Sea fish community and fisheries. Glob Chang Biol 13(7):1348-1367

Magath V, Thiel R (2013) Stock recovery, spawning period and spawning area expansion of the twaite shad Alosa fallax in the Elbe estuary, southern North Sea. Endanger Species Res 20:109-119

Malzahn AM, Boersma M (2012) Effects of poor food quality on copepod growth are dose dependent and non-reversible. Oikos 121:1408-1416

Margonski P, Hansson S, Tomczak MT, Grzebielec R (2010) Climate influence on Baltic cod, sprat, and herring stock-recruitment relationships. Progr Ocean 87:277-288

Martens P, van Beusekom JEE (2008) Zooplankton response to a warmer northern Wadden Sea. Helgoland Mar Res 62:67-75

Mathieson S, Cattrijsse A, Costa MJ, Drake P, Elliott M, Gardner J, Marchand J (2000) Fish assemblages of European tidal marshes: a comparison based on species, families and functional guilds. Mar Ecol Prog Ser 204:225-242

McLusky DS, Hull SC, Elliott M (1993) Variations in the intertidal and subtidal macrofauna and sediments along a salinity gradient in the upper Forth Estuary. Netherland J Aquat Ecol 27:101-109
Meier HEM (2015) Projected change - marine physics. In: The BACC II author team second assessment of climate change for the baltic sea basin. Regional Climate Studies. Springer, Heidelberg, New York, Dordrecht, London Meier HEM, Andersson HC, Eilola K, Gustafsson BG, Kuznetsov I, Müller-Karulis B, Neumann T, Savchuk OP (2011) Hypoxia in future climates: a model ensemble study for the Baltic Sea. Geophys Res Lett 38(24):L24608

Meier HEM, Hordoir R, Andersson HC, Dieterich C, Eilola K, Gustafsson BG, Höglund A, Schimanke $S$ (2012a) Modeling the combined impact of changing climate and changing nutrient loads on the Baltic Sea environment in an ensemble of transient simulations for 1961-2099. Clim Dynam 39(9):24212441

Meier HEM, Müller-Karulis B, Andersson HC, Dieterich C, Eilola K, Gustafsson BG, Höglund A, Hordoir R, Kuznetsov I, Neumann T, Ranjbar Z, Savchuk OP, Schimanke S (2012b) Impact of climate change on ecological quality indicators and biogeochemical fluxes in the Baltic Sea: a multi-model ensemble study. AMBIO 41(6):558-573

Menzel L, Bürger G (2002) Climate change scenarios and runoff response in the Mulde catchment (Southern Elbe, Germany). J Hydrol 267:53-64

Meyer PF (1930) Beobachtungen über das Auftreten von Sardellenschwärmen im Jadebusen 1931. Der Fischerbote 24.

Möller H (1984) Daten zur Biologie der Elbfische. Möller Verlag, Kiel

Möller H (1988) Fischbestande und Fischkrankheiten in der Unterelbe 19841986. Möller Verlag, Kiel

Möllmann C, Diekmann R (2012) Marine ecosystem regime shifts induced by climate and overfishing: a review for the northern hemispere. Adv Ecol Res 47:303-347

Möllmann C, Diekmann R, Müller-Karulis B, Kornilovs G, Plikshs M, Axe P (2009) Reorganization of a large marine ecosystem due to atmospheric and anthropogenic pressure: a discontinuous regime shift in the central Baltic Sea. Glob Change Biol 15:1377-1393

Moore KA, Jarvis JC (2008) Environmental factors affecting recent summertime eelgrass diebacks in the Lower Chesapeake Bay: implications for long-term persistence. J Coast Res Spec Issue 55:135-147

Mossbauer M, Dahlke S, Friedland R, Schernewski G (2013) Consequences of climate change and environmental policy for macroalgae accumulations on beaches along the German Baltic coastline. In: Schmidt-Thome P, Klein $J(H r s g)$ Climate change adaptation in practice: from strategy development to implementation. Wiley, Chichester, S 215-224

Nehring S (2006) Four arguments why so many alien species settle into estuaries, with special reference to the German river Elbe. Helgol Mar Res 60:127-134

Nehring S, Leuchs H (1999) The BfG-Monitoring in the German North Sea estuaries: macrozoobenthos. Senck Marit 29:107-111

Neumann H, Kröncke I (2011) The effect of temperature variability on ecological functioning of epifauna in the German Bight. Mar Ecol 32:49-57

Neumann H, Reiss H, Rakers S, Ehrich S, Kröncke I (2009) Temporal variability of southern North Sea epifauna communities after the cold winter 1995/1996. ICES J Mar Sci 66:2233-2243

Neumann H, de Boois I, Kröncke I, Reiss H (2013a) Climate change facilitated the range expansion of the non-native angular crab Goneplax rhomboides (Linnaeus, 1758) into the North Sea. Mar Ecol Prog Ser 484:143-153

Neumann H, Reiss H, Ehrich S, Sell A, Panten K, Kloppmann M, Wilhelms I, Kröncke I (2013b) Benthos and demersal fish habitats in the German Exclusive Economic Zone (EEZ) of the North Sea. Helgoland Mar Res 67:445-459

Neumann T (2010) Climate-change effects on the Baltic Sea ecosystem: a model study. J Mar Syst 81(3):213-224

Neumann T, Friedland R (2011) Climate change impacts on the Baltic Sea. In: Schernewski G, Hofstede J, Neumann T (Hrsg) Global change and baltic coastal zones. Springer, Dordrecht, S 23-32

Neumann T, Eilola K, Gustafsson B, Müller-Karulis B, Kuznetsov I, Meier HEM, Savchuk OP (2012) Extremes of temperature, oxygen and blooms in the Baltic Sea in a changing climate. AMBIO 41(6):574-585

Niiranen S, Yletyinen J, Tomczak MT, Blenckner T, Hjerne O, MacKenzie BR, Müller-Karulis B, Neumann T, Meier HEM (2013) Combined effects of global climate change and regional ecosystem drivers on an exploited marine food web. Glob Change Biol 19(11):3327-3342

Omstedt A, Edman M, Claremar B, Frodin P, Gustafsson E, Humborg C, Hägg H, Mörth M, Rutgersson A, Schurgers G, Smith B, Wällstedt T, Yurova A (2012) 
Future changes in the Baltic Sea acid-base $(\mathrm{pH})$ and oxygen balances. Tellus B 64:19586

Orendt C, Schmitt C, van Liefferinge C, Wolfram G, de Deckere E (2010) Include or exclude? A review on the role and suitability of aquatic invertebrate neozoa as indicators in biological assessment with special respect to fresh and brackish European waters. Biol Invasions 12:265-283

Österblom H, Hansson S, Larsson U, Hjerne O, Wulff F, Elmgren R, Folke C (2007) Human-induced trophic cascades and ecological regime shifts in the Baltic Sea. Ecosystems 10:877-889

Otto SA, Diekmann R, Flinkman J, Kornilovs G, Möllmann C (2014) Habitat heterogeneity determines climate impact on zooplankton community structure and dynamics. PLOS ONE 9:e90875

Paerl HW, Paul VJ (2012) Climate change: Links to global expansion of harmful cyanobacteria. Water Res 46(5):1349-1363

Peltonen H, Varjopuro R, Viitasalo M (2012) Climate change impacts on the Baltic Sea fish stocks and fisheries: review with a focus on Central Baltic herring, sprat and cod. Coastline Reports 35

Perry AL, Low PJ, Ellis JR, Reynolds JD (2005) Climate change and distribution shifts in marine fishes. Science 308:1912-1915

Philippart K, Epping E (2009) Climate change and ecology. Quality Status Report of the Wadden Sea (Webseiten des CWSS (international Wattenmeersekretariat) in Wilhelmshaven)

Philippart CJM, Beukema JJ, Cadée GC, Dekker R, Goedhart PW, von Iperen JM, Leopold MF, Herman PMJ (2007) Impact of nutrients on coastal communities. Ecosystems 10:95-118

Polte $\mathrm{P}$, Asmus $\mathrm{H}$ (2006a) Intertidal seagrass beds (Zostera noltii) as spawning grounds for transient fishes in the Wadden Sea. Mar Ecol Prog Ser 312:235243

Polte P, Asmus H (2006b) Influence of seagrass beds (Zostera noltii) on the species composition of juvenile fishes temporarily visiting the intertidal zone of the Wadden Sea. J Sea Res 55:244-252

Polte P, Schanz A, Asmus H (2005) The contribution of seagrass beds (Zostera noltii) to the function of tidal flats as a juvenile habitat for dominant, mobile epibenthos in the Wadden Sea. Mar Biol 147:813-822

Polte P, Kotterba P, Hammer C, Gröhsler T (2013) Survival bottlenecks in the early ontogenesis of Atlantic herring (Clupea harengus, L.) in coastal lagoon spawning areas of the western Baltic Sea. Ices J Mar Sci 71(4):982-990

Pörtner HO, Knust R (2007) Climate change affects marine fishes through the oxygen limitation of thermal tolerance. Science 315:95-97

Prena J, Gosselck F, Schroeren V, Voss J (1997) Periodic and episodic benthos recruitment in southwest Mecklenburg Bay (western Baltic Sea). Helgol Meeresunters 51(1):1-21

RADOST (2014) RADOST-Abschlussbericht. RADOST-Berichtsreihe Nr. 27. Ecologic Institute, Berlin

Reise K, Baptist M, Burbridge P, Dankers N, Fischer L, Flemming B, Oost A, Smit C (2010) The Wadden Sea-a universally outstanding tidal wetland. The Wadden Sea 2010. Common Wadden Sea Secretariat (CWSS). Trilateral Monitoring and Assessment Group, Wilhelmshaven (Wadden Sea Ecosystem 29)

Rick JJ, Kraberg AC, Asmus R, Wiltshire KH (2015) Phytoplankton and microzooplankton abundance in the Wadden Sea off List, Sylt. North Sea 2011-2013 Pangaea data base.

Rijnsdorp AD, Peck MA, Engelhard GH, Möllmann C, Pinnegar JK (2009) Resolving the effect of climate change on fish populations. ICES J Mar Sci 66:1570-1583

Rijnsdorp AD, Möllmann C, Peck MA, Engelhard GH, Pinnegar JK (2010) Synthesis and Working Hypotheses. In: Rijnsdorp AD, Möllmann C, Peck MA Engelhard GH, Pinnegar JK (Hrsg) Resolving climate impacts on fish stocks. ICES CRR 301:208-221

Scharfe M (2013) Analyse biologischer Langzeitveränderungen auf Basis hydroklimatischer Parameter in der südlichen Nordsee. Dissertation Universität Hamburg

Scharfe M, Callies U, Blöcker G, Petersen W, Schroeder F (2009) A simple Lagrangian model to simulate temporal variability of algae in the Elbe River. Ecol Model 220:2173-2186

Schiele KS, Darr A, Zettler M (2014) Verifying a biotope classification using benthic communities - an analysis towards the implementation of the European Marine Strategy Framework Directive. Mar Pollut Bull 78(12):181-189
Schlüter MJ, Merico A, Wiltshire KH, Greve W, von Storch H (2008) A statistical analysis of climate variability and ecosystem response in the German Bight. Ocean Dynam 58:169-186

Schlüter MH, Kraberg A, Wiltshire KH (2012) Long-term changes in the seasonality of selected diatoms related to grazers and environmental conditions. J Sea Res 67:91-97

Schöl A, Hein B, Wyrwa J, Kirchesch V (2014) Modelling water quality in the Elbe and its estuary - Large scale and long term applications with focus on the oxygen budget of the estuary. Küste 81:203-232

Schönberg W, Butzeck C, Eick D, Jensen K, Magath V, Thiel R, Rottgart E, Runge K (2014) Lebensraum Elbeästuar - auch 2050 alles im Fluss? In: KLIMZUGNORD Verbund (Hrsg) Kursbuch Klimaanpassung. Handlungsoptionen für die Metropolregion Hamburg. TuTech, Hamburg, S 96-97

Schückel U, Kröncke I (2013) Temporal changes in intertidal macrofauna communities over 8 decades: a result of eutrophication and climate change. Estuar Coast Shelf Sci 117:210-218

Schückel U, Beck M, Kröncke I (2013) Spatial variability in structural and functional aspects of macrofauna communities and their environmental parameters in the Jade Bay (Wadden Sea Lower Saxona, southern North Sea). Helgol Mar Res 67:121-136

Schückel U, Kröncke I, Baird D (2015) Linking long-term changes in trophic structure and function of an intertidal macrobenthic system to eutrophication and climate change using ecological network analysis. Mar Ecol Prog Ser 536:25-38

Sell A, Heessen H (2015) 46. Gurnards (Triglidae). In: Heessen H, Daan N, Ellis JR (Hrsg) Fish atlas of the Celtic Sea, North Sea, and Baltic Sea: based on international research-vessel surveys. Academic Publ, Wageningen, S 289-302

Sommer U, Aberle N, Lengfellner K, Lewandowska A (2012) The Baltic Sea spring phytoplankton bloom in a changing climate: an experimental approach. Mar Biol 159(11):2479-2490

Stal LJ, Staal M, Villbrandt M (1999) Nutrient control of cyanobacterial blooms in the Baltic Sea. Aquat Microb Ecol 18(2):165-173

Struyf E, van Damme S, Meire P (2004) Possible effects of climate change on estuarine nutrient fluxes: a case study in the highly nutrified Schelde estuary (Belgium, The Netherlands). Estuar Coast Shelf Sci 60:649-661

Taupp T, Wetzel MA (2013) Relocation of dredged material in estuaries under the aspect of the Water Framework Directive - a comparison of benthic quality indicators at dumping areas in the Elbe estuary. Ecol Indic 34:323-331

Taupp T, Wetzel MA (2014) Leaving the beaten track - approaches beyond the Venice System to classify estuarine waters according to salinity. Estuar Coast Shelf Sci 148:27-35

Thiel R (2011) Die Fischfauna europäischer Ästuare. Eine Strukturanalyse mit Schwerpunkt Tideelbe. Abhandlungen des Naturwissenschaftlichen Vereins Hamburg 43. Dölling \& Galitz, München, Hamburg, S 1-157

Thiel R (2014) Wie geht es Finte, Stint \& Co? Aktuelles über Zustand und zukünftige Entwicklung der Fischfauna in der Tideelbe. Nat Wissen 11:11-13

Thiel R, Potter IC (2001) The ichthyofaunal composition in the Elbe estuary: an analysis in space and time. Mar Biol 138(3):603-616

Thiel R, Thiel R (2015) Atlas der Fische und Neunaugen Hamburgs. Freie und Hansestadt Hamburg, Behörde für Stadtentwicklung und Umwelt, Hamburg

Thiel R, Sepúlveda A, Kafemann R, Nellen W (1995) Environmental factors as forces structuring the fish community of the Elbe estuary. J Fish Biol 46:47-69

Thiel R, Cabral H, Costa MJ (2003) Composition, temporal changes and ecological guild classification of the ichthyofaunas of large European estuaries - a comparison between the Tagus (Portugal) and the Elbe (Germany). J Appl Ichthyol 19:330-342

Thiel R, Riel P, Neumann R, Winkler HM, Böttcher U, Gröhsler T (2008) Return of twaite shad Alosa fallax (Lacépède, 1803) to the Southern Baltic Sea and the transitional area between the Baltic and North Seas. Hydrobiologia 602:161-177

Tian T, Su J, Flöser G, Wiltshire KH, Wirtz K (2011) Factors controlling the onset of spring blooms in the German Bight 2002-2005: light, wind and stratification. Cont Shelf Res 31:1140-1148

van der Veer HW, Dapper R, Henderson PA, Jung AS, Philippart CJM, Witte JIJ, Zuur AF (2015) Changes over 50 years in fish fauna of a temperate coastal sea: degradation of trophic structure and nursery function. Estuar Coast Shelf Sci 155:156-166

Wahl M, Jormalainen V, Eriksson BK, Coyer JA, Molis M, Schubert H, Dethier M, Karez R, Kruse I, Lenz M, Pearson G, Rohde S, Wikström SA, Olsen JL (2011) 
Stress ecology in fucus: abiotic, biotic and genetic interactions. In: Lesser (Hrsg) Advances in marine biology. Academic Press, Oxford, S 37-106

Wasmund N, Pollehne F, Postel L, Siegel H, Zettler ML (2011a) Biologische Zustandseinschätzung der Ostsee im Jahre 2010. Meereswissenschaftliche Berichte 85. Institut für Ostseeforschung, Warnemünde

Wasmund N, Tuimala J, Suikkanen S, Vandepitte L, Kraberg A (2011b) Long-term changes in phytoplankton composition in the western and central Baltic Sea. J Mar Syst 87:145-159

Wetzel MA, von der Ohe PC, Manz W, Koop JHE, Wahrendorf DS (2012) The ecological quality status of the Elbe estuary. A comparative approach on different benthic biotic indices applied to a highly modified estuary. Ecol Indic 19:118-129

Wetzel MA, Wahrendorf DS, von der Ohe PC (2013) Sediment pollution in the Elbe estuary and its potential toxicity at different trophic levels. Sci Total Environ 449:199-207

Wetzel MA, Scholle J, Teschke K (2014) Artificial structures in sediment-dominated estuaries and their possible influences on the ecosystem. Mar Environ Res 99:125-135

Wiltshire KH, Boersma M (2016) Meeting in the middle: on the interactions between microalgae and their predators or zooplankton and their food. In: Gilbert PM, Kana TM (Hrsg) Aquatic nutrient biogeochemistry and microbial ecology: a dual perspective. Springer, Berlin, S 215-223

Wiltshire KH, Kraberg A, Bartsch I, Boersma M, Franke HD, Freund J, Gebuhr C, Gerdts G, Stockmann K, Wichels A (2010) Helgoland Roads, North Sea: 45 years of change. Estuaries Coasts 33:295-310

Wiltshire KH, Boersma M, Carstens K, Kraberg AC, Peters S, Scharfe M (2015) Control of phytoplankton in a shelf sea: determination of the main drivers based on the Helgoland Roads Time Series. J Sea Res 105:42-52

Winder M, Sommer U (2012) Phytoplankton response to a changing climate. Hydrobiologia 698:5-16

Witte S, Buschbaum C, van Beusekom JEE, Reise K (2010) Does climatic warming explain why an introduced barnacle finally takes over after a lag of more than 50 years? Biol Invasions 12:3597-3589

Wolff WJ, Bakker JP, Laursen K, Reise K (2010) The wadden sea quality status report - synthesis report 2010. Wadden Sea Ecosystem 29. Common Wadden Sea Secretariat (CWSS), Wilhelmshaven, S 25-74

Zettler ML, Bönsch R, Gosselck F (2000) Verbreitung des Makrozoobenthos in der Mecklenburger Bucht (südliche Ostsee) - rezent und im historischem Vergleich. Meereswissenschaftliche Berichte Institut für Ostseeforschung, Warnemünde

Open Access Dieses Kapitel wird unter der Creative Commons Namensnennung - Nicht kommerziell 4.0 International Lizenz (http://creativecommons.org/licenses/by-nc/4.0/deed.de) veröffentlicht, welche die nicht-kommerzielle Nutzung, Vervielfältigung, Bearbeitung, Verbreitung und Wiedergabe in jeglichem Medium und Format erlaubt, sofern Sie den/die ursprünglichen Autor(en) und die Quelle ordnungsgemäß nennen, einen Link zur Creative Commons Lizenz beifügen und angeben, ob Änderungen vorgenommen wurden.

Die in diesem Kapitel enthaltenen Bilder und sonstiges Drittmaterial unterliegen ebenfalls der genannten Creative Commons Lizenz, sofern sich aus der Abbildungslegende nichts anderes ergibt. Sofern das betreffende Material nicht unter der genannten Creative Commons Lizenz steht und die betreffende Handlung nicht nach gesetzlichen Vorschriften erlaubt ist, ist auch für die oben aufgeführten nicht-kommerziellen Weiterverwendungen des Materials die Einwilligung des jeweiligen Rechteinhabers einzuholen. 